\title{
ON PROPERTIES OF THE GENERALIZED MAJORIZATION*
}

\author{
MARIJA DODIG ${ }^{\dagger}$ AND MARKO STOŠIĆ $\ddagger$
}

\begin{abstract}
In this paper, a complete solution of a problem involving generalized majorization of partitions is given: for two pairs of partitions $(\mathbf{d}, \mathbf{a})$ and $(\mathbf{c}, \mathbf{b})$ necessary and sufficient conditions for the existence of a partition $\mathbf{g}$ that is majorized by both pairs is determined. The obtained conditions are explicit, the solution is constructive and it uses novel techniques and indices. Although the problem is motivated by the applications in matrix pencil completions problems, all results are purely combinatorial and they give a new perspective on comparison of partitions.
\end{abstract}

Key words. Partitions, Generalized majorization, Matrix completion.

AMS subject classifications. 05A17, 15A83.

1. Introduction. The concept of majorization of partitions turned out to be a powerful tool in matrix and matrix pencils completion problems.

Let $\mathbf{a}:=\left(a_{1}, \ldots, a_{s}\right)$ and $\mathbf{w}:=\left(w_{1}, \ldots, w_{s}\right)$ be two partitions, i.e., two finite non-increasing sequences of integers. If

$$
\sum_{i=1}^{j} w_{i} \leq \sum_{i=1}^{j} a_{i}, \text { for all } j=1, \ldots, s,
$$

and

$$
\sum_{i=1}^{s} w_{i}=\sum_{i=1}^{s} a_{i}
$$

then we say that $\mathbf{w}$ is majorized by $\mathbf{a}$, and write

$$
\mathbf{w} \prec \mathbf{a} .
$$

This, classical, majorization is well studied and there are many results concerning its properties [3, 12, 13, 16. It also appears in matrix completion problems, see e.g., [2, 11, 17, 18, in particular, to express conditions for matrix row completion problem up to a square matrix.

*Received by the editors on December 5, 2012. Accepted for publication on May 26, 2013. Handling Editor: Shmuel Friedland.

${ }^{\dagger}$ Centro de Estruturas Lineares e Combinatórias, CELC, Universidade de Lisboa, Av. Prof. Gama Pinto 2, 1649-003 Lisbon, Portugal (dodig@cii.fc.ul.pt).

$\ddagger$ Instituto de Sistemas e Robótica and CAMGSD, Instituto Superior Técnico, Av. Rovisco Pais 1, 1049-001 Lisbon, Portugal. 
However, by using classical majorization one cannot compare more than two partitions. For that reason in [4] the notion generalized majorization was introduced. It deals with triples of partitions $\mathbf{a}, \mathbf{d}$ and $\mathbf{g}$, such that the length of $\mathbf{g}$ is equal to the sum of the lengths of $\mathbf{a}$ and $\mathbf{d}$. The generalized majorization is denoted by

$$
\mathbf{g} \prec^{\prime}(\mathbf{d}, \mathbf{a}) .
$$

In the case when $\mathbf{d}$ is a partition of length zero, the generalized majorization becomes the classical majorization between partitions $\mathbf{g}$ and $\mathbf{a}$.

This majorization was motivated by results in matrix completion problems in the case of column or row completion of rectangular matrices. As to our knowledge, it first appeared in [1, 15], and later on in [5, 8, 9]. In [9] some combinatorial properties of the generalized majorization have been obtained, including the generalization of the elementary operations for classical majorization.

The generalized majorization turns out to be a very convenient way of writing the conditions of the results in completion problems involving both row and column minimal indices.

In this paper, we give a complete solution to the following problem:

Problem 1. Let $\mathbf{a}, \mathbf{b}, \mathbf{c}$ and $\mathbf{d}$ be partitions such that the sum of the lengths of $\mathbf{a}$ and $\mathbf{d}$ is equal to the sum of the lengths of $\mathbf{b}$ and $\mathbf{c}$.

Find necessary and sufficient conditions for the existence of a partition $\mathbf{g}$, such that

$$
\begin{aligned}
& \mathrm{g} \prec^{\prime}(\mathbf{d}, \mathbf{a}) \\
& \mathrm{g} \prec^{\prime}(\mathbf{c}, \mathbf{b}) .
\end{aligned}
$$

Problem 1 is a hard and challenging combinatorial task. The obtained conditions involve novel indices and labels on the partitions $\mathbf{c}$ and $\mathbf{d}$ introduced in Section 3 . In fact, Problem 1 was inspired by matrix and matrix pencils completion problems. Its solution enables new approach to those problems, since it allows studying relations between partitions made of column (or row) minimal indices of the pencils involved. For instance, a solution of a particular case of Problem 1 when $\mathbf{b}=0$ was obtained in $[5,8$.

We expect more applications of this result. In particular, its impact on the general rank distance problem [7, as well as to the general matrix pencils completion problem [14] and its particular cases [6, 7, 10, is crucial. 
1.1. Notation. For any sequence of integers satisfying $c_{1} \geq \cdots \geq c_{m}$, by $\sum_{c_{i} \leq a} c_{i}$ we mean the sum of all the integers $c_{i}$ that are less than or equal to $a$. We put $\sum_{i \in W} c_{i}=0$ whenever $W$ is an empty set. Also, we assume that $\sum_{i=a}^{b} c_{i}=0$ if $a>b$. Finally, we assume $c_{0}=+\infty$ and $c_{m+1}=c_{m+2}=\cdots=-\infty$.

Let $x$ be an integer such that $c_{j} \geq x \geq c_{j+1}$, for some $j \in\{0, \ldots, m\}$. And let $\mathbf{c}=$ $\left(c_{1}, \ldots, c_{m}\right)$. Then by $\mathbf{c} \cup\{x\}$ we mean the partition $\overline{\mathbf{c}}=\left(c_{1}, \ldots, c_{j}, x, c_{j+1}, \ldots, c_{m}\right)$. Also, $\overline{\mathbf{c}} \backslash\{x\}:=\mathbf{c}$.

2. Generalized majorization. Generalized majorization presents a generalization of the majorization in Hardy-Littlewood-Pólya sense 12. It deals with three partitions such that the length of one of them is equal to the sum of the lengths of another two.

Let $m$ and $s$ be nonnegative integers, and let $d_{1} \geq \cdots \geq d_{m}, g_{1} \geq \cdots \geq g_{m+s}$, and $a_{1} \geq \cdots \geq a_{s}$ be integers.

Consider partitions

$$
\begin{aligned}
& \mathbf{a}=\left(a_{1}, \ldots, a_{s}\right), \\
& \mathbf{d}=\left(d_{1}, \ldots, d_{m}\right)
\end{aligned}
$$

and

$$
\mathbf{g}=\left(g_{1}, \ldots, g_{m+s}\right)
$$

DeFinition 2.1. If

$$
\begin{gathered}
d_{i} \geq g_{i+s}, \quad i=1, \ldots, m, \\
\sum_{i=1}^{h_{j}} g_{i}-\sum_{i=1}^{h_{j}-j} d_{i} \leq \sum_{i=1}^{j} a_{i}, \quad j=1, \ldots, s, \\
\sum_{i=1}^{m+s} g_{i}=\sum_{i=1}^{m} d_{i}+\sum_{i=1}^{s} a_{i},
\end{gathered}
$$

where $h_{j}:=\min \left\{i \mid d_{i-j+1}<g_{i}\right\}, j=1, \ldots, s$, then we say that $\mathbf{g}$ is majorized by $\mathbf{d}$ and a. This type of majorization we call the generalized majorization, and we write

$$
\mathbf{g} \prec^{\prime}(\mathbf{d}, \mathbf{a}) .
$$

As to our knowledge, this type of majorization was first considered in [1, 15], and later on under this name in [4, 5, 6, 8, 9]. 
If $m=0$, i.e., if $\mathbf{d}$ is an empty partition, then the generalized majorization between $\mathbf{g}$ and $(\mathbf{d}, \mathbf{a})$, becomes a classical majorization between $\mathbf{g}$ and $\mathbf{a}$.

We note that, if (2.6) is satisfied, then (2.5) is equivalent to the following:

$$
\sum_{i=h_{j}+1}^{m+s} g_{i} \geq \sum_{i=h_{j}-j+1}^{m} d_{i}+\sum_{i=j+1}^{s} a_{i}, \quad j=1, \ldots, s .
$$

Also, we note that from the definition of $h_{j}$ 's we have:

$$
0<h_{1}<h_{2}<\cdots<h_{s}<m+s+1
$$

and we set $h_{0}:=0$ and $h_{s+1}:=m+s+1$.

There is an additional property given in [8, Lemma 4.2]:

LEMMA 2.2. Suppose that $d_{1} \geq \cdots \geq d_{m}, g_{1} \geq \cdots \geq g_{m+s}$ and $a_{1} \geq \cdots \geq a_{s}$ satisfy (2.4) and (2.7). Let $u$ be such that $h_{j}<u \leq h_{j+1}$, for some $j \in\{0, \ldots, s\}$. Then the following is also valid:

$$
\sum_{i=u}^{m+s} g_{i} \geq \sum_{i=u-j}^{m} d_{i}+\sum_{i=j+1}^{s} a_{i}
$$

Various combinatorial properties of the generalized majorization have been obtained in 9. In this paper, we are focusing on different properties and aspects of generalized majorization.

2.1. Basic properties of the generalized majorization. In this subsection, we show some properties of generalized majorization that enables simplifying partitions involved. More precisely, the results of the following lemmas will allow that without loss of generality Problem 1 can be considered in the case when the partitions c and $\mathbf{d}$ do not have the same elements.

Lemma 2.3. Let $\mathbf{a}, \mathbf{d}$ and $\mathbf{g}$ be partitions as given in (2.1)-(2.3). Let $x$ be an integer and let $\mathbf{g}^{\prime}:=\mathbf{g} \cup\{x\}$ and $\mathbf{d}^{\prime}:=\mathbf{d} \cup\{x\}$. Then

$$
\mathbf{g} \prec^{\prime}(\mathbf{d}, \mathbf{a})
$$

if and only if

$$
\mathbf{g}^{\prime} \prec^{\prime}\left(\mathbf{d}^{\prime}, \mathbf{a}\right) \text {. }
$$


Proof. Let $u=\max \left\{i \mid g_{i}>x\right\}$. Then

$$
g_{u}>x \geq g_{u+1} .
$$

Let $l=\max \left\{i \mid d_{i}>x\right\}$. Then

$$
d_{l}>x \geq d_{l+1} .
$$

Let $\mathbf{g}^{\prime}=\left(g_{1}^{\prime}, \ldots, g_{m+s+1}^{\prime}\right)$ and $\mathbf{d}^{\prime}=\left(d_{1}^{\prime}, \ldots, d_{m+1}^{\prime}\right)$. From (2.10) and (2.11) we have

$$
\begin{array}{ll}
\mathbf{g}^{\prime}: & g_{1} \geq \cdots \geq g_{u}>x \geq g_{u+1} \geq \cdots \cdots \\
\mathbf{d}^{\prime}: & d_{1} \geq \cdots \geq d_{l}>\quad x \geq d_{l+1} \geq \cdots \geq d_{m+s},
\end{array}
$$

i.e.,

$$
\begin{aligned}
& g_{i}^{\prime}=g_{i}, \quad i \leq u, \\
& g_{u+1}^{\prime}=x, \quad d_{l+1}^{\prime}=x, \\
& g_{i}^{\prime}=g_{i-1}, \quad i \geq u+2, \quad d_{i}^{\prime}=d_{i-1}, \quad i \geq l+2 .
\end{aligned}
$$

Let $h_{j}:=\min \left\{i \mid d_{i-j+1}<g_{i}\right\}, j=1, \ldots, s\left(h_{0}:=0, h_{s+1}:=m+s+1\right)$, and $h_{j}^{\prime}:=\min \left\{i \mid d_{i-j+1}^{\prime}<g_{i}^{\prime}\right\}, j=1, \ldots, s\left(h_{0}^{\prime}:=0, h_{s+1}^{\prime}:=m+s+2\right)$.

Let $\alpha \in\{0, \ldots, s\}$ be such that $h_{\alpha} \leq u<h_{\alpha+1}$, i.e., such that $g_{h_{\alpha}}>x \geq g_{h_{\alpha}+1}$. Then by the definition of $h_{\alpha}$ and $h_{\alpha+1}$, we have

$$
d_{h_{\alpha}-\alpha} \geq g_{h_{\alpha}-1} \geq g_{h_{\alpha}}>x \geq g_{h_{\alpha+1}}>d_{h_{\alpha+1}-\alpha} .
$$

By (2.12), (2.13) implies $u \leq l+\alpha$, and thus we have

$$
\begin{gathered}
h_{j}^{\prime}=h_{j}, \quad j \leq \alpha, \\
h_{j}^{\prime}=h_{j}+1, \quad j>\alpha .
\end{gathered}
$$

Now, suppose that (2.8) is valid. From the definition of the generalized majorization, we have that (2.8) is equivalent to (2.4), (2.5) and (2.6).

In order to prove (2.9), we are left with proving

$$
\begin{gathered}
d_{i}^{\prime} \geq g_{i+s}^{\prime}, \quad i=1, \ldots, m+1, \\
\sum_{i=1}^{h_{j}^{\prime}} g_{i}^{\prime}-\sum_{i=1}^{h_{j}^{\prime}-j} d_{i}^{\prime} \leq \sum_{i=1}^{j} a_{i}, \quad j=1, \ldots, s, \\
\sum_{i=1}^{m+s+1} g_{i}^{\prime}=\sum_{i=1}^{m+1} d_{i}^{\prime}+\sum_{i=1}^{s} a_{i} .
\end{gathered}
$$

Equation (2.18) follows directly by (2.6) and by the definition of $\mathbf{g}^{\prime}$ and $\mathbf{d}^{\prime}$. 
Let us prove (2.16). By (2.4) and (2.11), we have $x \geq d_{l+1} \geq g_{l+1+s}$. Hence, by (2.10) we obtain

$$
u \leq l+s .
$$

By (2.4), (2.19) and (2.12), for $i \geq l+2$ we have

$$
d_{i}^{\prime}=d_{i-1} \geq g_{i-1+s}=g_{i+s}^{\prime} .
$$

For $i \leq u-s$, we have

$$
d_{i}^{\prime}=d_{i} \geq g_{i+s}=g_{i+s}^{\prime}
$$

Finally, for $u-s+1 \leq i \leq l+1$, we have

$$
d_{i}^{\prime} \geq d_{l+1}^{\prime}=x=g_{u+1}^{\prime} \geq g_{i+s}^{\prime} .
$$

Hence, we have proved that (2.16) is valid.

Now we are left with proving (2.17). Since $h_{\alpha} \leq u<h_{\alpha+1}$, (2.17) for $j \leq \alpha$, becomes the same inequality as in (2.5), while for $j>\alpha$, (2.17) has the same additional summand $x$ added to the both sides of (2.5). Hence, (2.17) follows by (2.5).

Now suppose that (2.9) is valid.

In order to prove (2.8) we are left with proving (2.4), (2.5) and (2.6). Since (2.9) is valid, we have (2.16), (2.17) and (2.18). Hence, the definitions of $\mathbf{g}$ and $\mathbf{d}$ together with (2.18) give (2.6)

By (2.16), we have that $x=d_{l+1}^{\prime} \geq g_{l+s+1}^{\prime}$, and so

$$
u \leq l+s .
$$

Then, we have

$$
\begin{array}{cc}
\text { for } i \geq l+1: & d_{i}=d_{i+1}^{\prime} \geq g_{i+1+s}^{\prime}=g_{i+s}, \\
\text { for } i \leq u-s: & d_{i}=d_{i}^{\prime} \geq g_{i+s}^{\prime}=g_{i+s}, \\
\text { for } u-s<i \leq l: & d_{i} \geq d_{l}>x \geq g_{u+1} \geq g_{i+s}
\end{array}
$$

which proves (2.4).

Hence, we are left with proving (2.5). Now, for $j \leq \alpha$, from (2.14) the inequality (2.5) is the same as the corresponding inequality in (2.17). For $j>\alpha$, from (2.15), the inequality in (2.5) is the same as the corresponding inequality in (2.17) after subtracting the same summand, $x$, from both sides. Hence, (2.5) follows from (2.17), which finishes our proof.

Lemma 2.4. Let $\mathbf{a}=\left(a_{1}, \ldots, a_{s}\right), \mathbf{d}=\left(d_{1}, \ldots, d_{m}\right)$, and $\overline{\mathbf{g}}=\left(\bar{g}_{1}, \ldots, \bar{g}_{m+s}\right)$ be partitions such that

$$
d_{i} \geq \bar{g}_{i+s},
$$




$$
\begin{aligned}
\sum_{i=\bar{h}_{j}+1}^{m+s} \bar{g}_{i} \geq & \sum_{i=\bar{h}_{j}-j+1}^{m} d_{i}+\sum_{i=j+1}^{s} a_{i}, \quad j=1, \ldots, s, \\
& \sum_{i=1}^{m+s} \bar{g}_{i} \geq \sum_{i=1}^{m} d_{i}+\sum_{i=1}^{s} a_{i},
\end{aligned}
$$

where $\bar{h}_{j}:=\min \left\{i \mid d_{i-j+1}<\bar{g}_{i}\right\}, j=1, \ldots, s$.

Let $f \in\{2, \ldots, m+s\}$, and let $\mathbf{g}=\left(g_{1}, \ldots, g_{m+s}\right)$ be a partition such that

$$
\begin{gathered}
g_{i}=\bar{g}_{i}, \quad i \geq f, \\
g_{i} \leq \bar{g}_{i}, \quad i<f, \\
\bar{g}_{f-1} \geq g_{1} \geq g_{f-1} \geq g_{1}-1, \\
\sum_{i=1}^{m+s} g_{i} \geq \sum_{i=1}^{m} d_{i}+\sum_{i=1}^{s} a_{i} .
\end{gathered}
$$

Then

$$
\sum_{i=h_{j}+1}^{m+s} g_{i} \geq \sum_{i=h_{j}-j+1}^{m} d_{i}+\sum_{i=j+1}^{s} a_{i}, \quad j=0, \ldots, s,
$$

where $h_{j}:=\min \left\{i \mid d_{i-j+1}<g_{i}\right\}, j=1, \ldots, s, h_{0}:=0$.

Proof. From the definition of $h_{j}$ and $\bar{h}_{j}$ we have $\bar{h}_{j} \leq h_{j}, j=1, \ldots, s$. Moreover, let $p \in\{0, \ldots, s\}$ be such that $\bar{h}_{p}<f \leq \bar{h}_{p+1}$. Then we have that $h_{j}=\bar{h}_{j}, j \geq p+1$, and so by (2.20), equation (2.22) is trivially satisfied for all $j \geq p+1$.

Since $f-1<\bar{h}_{p+1}$, we have $d_{f-p-1} \geq \bar{g}_{f-1} \geq g_{1}$. Thus, $h_{j} \geq f-p+j-1$, for every $j=1, \ldots, p$.

We shall prove by induction on $j$ that the condition (2.22) is satisfied for every $j=0, \ldots, p$, thus completing the proof of (2.22).

If $j=0$, (2.22) equals (2.21).

Now, let $1 \leq j \leq p$, and suppose that (2.22) is satisfied for $j-1$.

If $g_{h_{j}} \leq a_{j}$, then by the induction hypothesis, we have

$$
\begin{aligned}
\sum_{i=h_{j}+1}^{m+s} g_{i} & =\sum_{i=h_{j-1}+1}^{m+s} g_{i}-\sum_{i=h_{j-1}+1}^{h_{j}} g_{i} \geq \sum_{i=h_{j-1}-j+2}^{m} d_{i}+\sum_{i=j}^{s} a_{i}-\sum_{i=h_{j-1}+1}^{h_{j}-1} g_{i}-g_{h_{j}}= \\
2.23) & =\sum_{i=h_{j}-j+1}^{m} d_{i}+\sum_{i=j+1}^{s} a_{i}+\sum_{i=h_{j-1}-j+2}^{h_{j}-j} d_{i}-\sum_{i=h_{j-1}+1}^{h_{j}-1} g_{i}+a_{j}-g_{h_{j}} .
\end{aligned}
$$


By the definition of $h_{j}$, we have that for all $i<h_{j}, g_{i} \leq d_{i-j+1}$. Hence, by (2.23) follows (2.22), as wanted.

So, in the rest of the proof of (2.22) we assume that $g_{h_{j}}>a_{j}$. Then since $g_{h_{j}} \leq g_{1} \leq g_{f-1}+1$, we have $g_{f-1}+1>a_{j}$, i.e., $g_{f-1} \geq a_{j}$.

If $h_{j}<f$, then by (2.20) and Lemma 2.2, we have

$$
\begin{aligned}
& \sum_{i=h_{j}+1}^{m+s} g_{i}=\sum_{i=h_{j}+1}^{f-1} g_{i}+\sum_{i=f}^{m+s} \bar{g}_{i} \geq \sum_{i=h_{j}+1}^{f-1} g_{i}+\sum_{i=f-p}^{m} d_{i}+\sum_{i=p+1}^{s} a_{i}= \\
& =\left(\sum_{i=h_{j}+1}^{f-1} g_{i}+\sum_{i=f-p}^{h_{j}-j} d_{i}-\sum_{i=j+1}^{p} a_{i}\right)+\left(\sum_{i=h_{j}-j+1}^{m} d_{i}+\sum_{i=j+1}^{s} a_{i}\right) .
\end{aligned}
$$

Let us see that $\sum_{i=h_{j}+1}^{f-1} g_{i}+\sum_{i=f-p}^{h_{j}-j} d_{i}-\sum_{i=j+1}^{p} a_{i} \geq 0$. Indeed, by the definition of $h_{j}$ we have

$$
\begin{aligned}
\sum_{i=h_{j}+1}^{f-1} g_{i}+\sum_{i=f-p}^{h_{j}-j} d_{i}-\sum_{i=j+1}^{p} a_{i} & \geq \sum_{i=h_{j}+1}^{f-1} g_{i}+\sum_{i=f-p+j-1}^{h_{j}-1} g_{i}-\sum_{i=j+1}^{p} a_{i} \\
& \geq \sum_{i=f-p+j}^{f-1} g_{i}-\sum_{i=j+1}^{p} a_{i},
\end{aligned}
$$

and since $g_{f-1} \geq a_{j}$, the last expression is nonnegative, as wanted.

If $h_{j} \geq f$, then $\bar{h}_{p}<f<h_{j}+1 \leq h_{p+1}=\bar{h}_{p+1}$. Thus, by (2.20) and Lemma 2.2, we have

$$
\begin{aligned}
\sum_{i=h_{j}+1}^{m+s} g_{i} & =\sum_{i=h_{j}+1}^{m+s} \bar{g}_{i} \geq \sum_{i=h_{j}+1-p}^{m} d_{i}+\sum_{i=p+1}^{s} a_{i} \\
& =\left(\sum_{i=h_{j}+1-p}^{h_{j}-j} d_{i}-\sum_{i=j+1}^{p} a_{i}\right)+\left(\sum_{i=h_{j}-j+1}^{m} d_{i}+\sum_{i=j+1}^{s} a_{i}\right) .
\end{aligned}
$$

Finally, since $g_{h_{j}}>a_{j}$, from the definition of $h_{j}$, we have

$$
\sum_{i=h_{j}+1-p}^{h_{j}-j} d_{i}-\sum_{i=j+1}^{p} a_{i} \geq \sum_{i=h_{j}-p+j}^{h_{j}-1} g_{i}-\sum_{i=j+1}^{p} a_{i} \geq 0
$$

as wanted.

LEMMA 2.5. Let $\mathbf{a}, \mathbf{d}$ and $\mathbf{g}$ be partitions as given in (2.1)-(2.3), such that

$$
\mathrm{g} \prec^{\prime}(\mathbf{d}, \mathbf{a}) .
$$


Let $x$ be an integer such that there exists $u \in\{1, \ldots, m\}$ with $d_{u}=x$, and there does not exist $l \in\{1, \ldots, m+s\}$ such that $g_{l}=x$.

Then there exists a partition $\mathbf{g}^{\prime}=\left(g_{1}^{\prime}, \ldots, g_{m+s}^{\prime}\right)$, such that

$$
\mathbf{g}^{\prime} \prec^{\prime}(\mathbf{d}, \mathbf{a})
$$

and such that there exists $l \in\{1, \ldots, m+s\}$ with $g_{l}^{\prime}=x$. Moreover, such a $\mathbf{g}^{\prime}$ can be defined independently from $\mathbf{d}$ and $\mathbf{a}$, i.e., it depends only on $\mathbf{g}$ and $x$.

Proof. First suppose that $g_{1}>x>g_{m+s}$. Then there exists $j \in\{1, \ldots, m+s-1\}$ such that

$$
g_{j}>x>g_{j+1}
$$

If $g_{j}+g_{j+1} \geq 2 x$, then let $g_{j}^{\prime}=g_{j}+g_{j+1}-x$ and $g_{j+1}^{\prime}=x$.

If $g_{j}+g_{j+1}<2 x$, then let $g_{j}^{\prime}=x$ and $g_{j+1}^{\prime}=g_{j}+g_{j+1}-x$.

In both cases, we have $g_{j} \geq g_{j}^{\prime} \geq g_{j+1}^{\prime} \geq g_{j+1}$. Also, for $i \neq j, j+1$, we set $g_{i}^{\prime}=g_{i}$. Thus, we have that $g_{i}^{\prime}$ 's are nonincreasing. We claim that for such defined $\mathbf{g}^{\prime}:=\left(g_{1}^{\prime}, \ldots, g_{m+s}^{\prime}\right)$ we have

$$
\mathbf{g}^{\prime} \prec^{\prime}(\mathbf{d}, \mathbf{a})
$$

By the definition of $\prec^{\prime}$, 2.25) is equivalent to:

$$
\begin{gathered}
d_{i} \geq g_{i+s}^{\prime}, \quad i=1, \ldots, m \\
\sum_{i=1}^{h_{w}^{\prime}} g_{i}^{\prime}-\sum_{i=1}^{h_{w}^{\prime}-w} d_{i} \leq \sum_{i=1}^{w} a_{i}, \quad w=1, \ldots, s \\
\sum_{i=1}^{m+s} g_{i}^{\prime}=\sum_{i=1}^{m} d_{i}+\sum_{i=1}^{s} a_{i}
\end{gathered}
$$

where $h_{w}^{\prime}:=\min \left\{i \mid d_{i-w+1}<g_{i}^{\prime}\right\}, w=1, \ldots, s$. Since (2.24) is valid, we have (2.4), (2.5) and (2.6). Hence, the definition of $\mathbf{g}^{\prime}$ together with (2.6), gives (2.28).

For all $i \neq j-s+1$, we have $d_{i} \geq g_{i+s} \geq g_{i+s}^{\prime}$.

Since $d_{j-s} \geq g_{j}>x=d_{u}$, we have $u \geq j-s+1$. So

$$
d_{j-s+1} \geq d_{u}=x \geq g_{j+1}^{\prime},
$$

as well. Hence, we have proved (2.26). 
Let $h_{w}:=\min \left\{i \mid d_{i-w+1}<g_{i}\right\}, w=1, \ldots, s\left(h_{0}:=0, h_{s+1}:=m+s+1\right)$. Now let $\alpha \in\{0, \ldots, s\}$ be such that

$$
h_{\alpha} \leq j<h_{\alpha+1}
$$

First of all, we have $j+1<h_{\alpha+1}$. Otherwise, we would have $j+1=h_{\alpha+1}$, and so $g_{j+1}=g_{h_{\alpha+1}}>d_{j+1-\alpha}$. However, since $j<h_{\alpha+1}$, we have $d_{j-\alpha} \geq g_{j}>x$, and so $u \geq j-\alpha+1$. Hence, $d_{j-\alpha+1} \geq d_{u}=x>g_{j+1}$, which is a contradiction.

Now, if $h_{\alpha}<j<j+1<h_{\alpha+1}$, then we have $d_{j-\alpha} \geq g_{j}>x$, and so $u \geq j-\alpha+1$. Hence, $d_{j-\alpha+1} \geq x \geq g_{j+1}^{\prime}$. So, in this case we have $h_{i}^{\prime}=h_{i}$, for all $i=1, \ldots, s$, and

$$
\sum_{i=1}^{h_{l}} g_{i}=\sum_{i=1}^{h_{l}^{\prime}} g_{i}^{\prime}, \quad l=1, \ldots, s
$$

Hence, (2.27) follows from (2.5).

If $h_{\alpha}=j<j+1<h_{\alpha+1}$, then again we have $d_{j-\alpha} \geq g_{j-1} \geq g_{j}>x$, and so $u \geq j-\alpha+1$, i.e., $d_{j-\alpha+1} \geq x \geq g_{j+1}^{\prime}$. So $h_{i}^{\prime}=h_{i}$, for $i \geq \alpha+1$. Also, obviously $h_{i}^{\prime}=h_{i}$, for $i \leq \alpha-1$. Since, for all $l \neq \alpha$ we have

$$
\sum_{i=1}^{h_{l}} g_{i}=\sum_{i=1}^{h_{l}^{\prime}} g_{i}^{\prime}
$$

for all such indices (2.27) follows from (2.5).

As for $h_{\alpha}^{\prime}$, we have $h_{\alpha} \leq h_{\alpha}^{\prime}<h_{\alpha+1}^{\prime}=h_{\alpha+1}$.

If $h_{\alpha}^{\prime}=h_{\alpha}$, then

$$
\sum_{i=1}^{h_{\alpha}^{\prime}} g_{i}^{\prime}=\sum_{i=1}^{h_{\alpha}} g_{i}^{\prime} \leq \sum_{i=1}^{h_{\alpha}} g_{i} \leq \sum_{i=1}^{h_{\alpha}-\alpha} d_{i}+\sum_{i=1}^{\alpha} a_{i}=\sum_{i=1}^{h_{\alpha}^{\prime}-\alpha} d_{i}+\sum_{i=1}^{\alpha} a_{i}
$$

If $h_{\alpha}^{\prime}>h_{\alpha}$, then

$\sum_{i=1}^{h_{\alpha}^{\prime}} g_{i}^{\prime}=\sum_{i=1}^{h_{\alpha}^{\prime}} g_{i}=\sum_{i=1}^{h_{\alpha}} g_{i}+\sum_{i=h_{\alpha}+1}^{h_{\alpha}^{\prime}} g_{i} \leq \sum_{i=1}^{h_{\alpha}-\alpha} d_{i}+\sum_{i=1}^{\alpha} a_{i}+\sum_{i=h_{\alpha}-\alpha+1}^{h_{\alpha}^{\prime}-\alpha} d_{i}=\sum_{i=1}^{h_{\alpha}^{\prime}-\alpha} d_{i}+\sum_{i=1}^{\alpha} a_{i}$.

The middle inequality follows from (2.5) for $j=\alpha$ and the fact that $h_{\alpha}^{\prime}<h_{\alpha+1}$. This ends the proof in the case $g_{1}>x>g_{m+s}$.

Since $x=d_{u} \geq d_{m} \geq g_{m+s}$, the only remaining case is

$$
x>g_{1} \text {. }
$$


In this case, let

$$
g_{1}^{\prime}=x
$$

and we define the rest of $g_{i}^{\prime}$ 's in the following way:

Let $f:=\max \left\{v \in\{2, \ldots, m+s\} \mid(v-1) g_{v}+x>\sum_{i=1}^{v} g_{i}\right\}$. Then we define $g_{i}^{\prime}=g_{i}$ for $i>f$, and we define $g_{2}^{\prime} \geq \cdots \geq g_{f}^{\prime}$ as the most homogeneous partition such that $\sum_{i=2}^{f} g_{i}^{\prime}=\sum_{i=1}^{f} g_{i}-x$.

Such defined $g_{1}^{\prime} \geq \cdots \geq g_{m+s}^{\prime}$ satisfy (2.28). Also, since $d_{i} \geq g_{i+s} \geq g_{i+s}^{\prime}$ for all $i=1, \ldots, m$, we have (2.26). So we are left with proving (2.27). Since $d_{1} \geq d_{u}=x=g_{1}^{\prime} \geq g_{1}$, we have that $h_{1} \geq 2$,

$$
\sum_{i=2}^{m+s} g_{i} \geq \sum_{i=2}^{m} d_{i}+\sum_{i=1}^{s} a_{i}
$$

and

$$
\sum_{i=2}^{m+s} g_{i}^{\prime} \geq \sum_{i=2}^{m} d_{i}+\sum_{i=1}^{s} a_{i}
$$

If we denote $\tilde{\mathbf{g}}=\mathbf{g} \backslash\left\{g_{1}\right\}, \tilde{\mathbf{d}}=\mathbf{d} \backslash\left\{d_{1}\right\}$ and $\tilde{\mathbf{g}}^{\prime}=\mathbf{g}^{\prime} \backslash\left\{g_{1}^{\prime}\right\}$, then by applying Lemma 2.4 for $\tilde{\mathbf{g}}, \tilde{\mathbf{d}}$, a and $\tilde{\mathbf{g}}^{\prime}$, we obtain (2.27), as wanted.

2.2. Relaxation on partitions $\mathbf{c}$ and $\mathbf{d}$. Let $m, n, k$ and $s$ be nonnegative integers such that

$$
m+s=n+k \text {. }
$$

Let

$$
\begin{gathered}
\mathbf{a}=\left(a_{1}, \ldots, a_{s}\right), \\
\mathbf{b}=\left(b_{1}, \ldots, b_{k}\right), \\
\mathbf{c}=\left(c_{1}, \ldots, c_{n}\right)
\end{gathered}
$$

and

$$
\mathbf{d}=\left(d_{1}, \ldots, d_{m}\right)
$$

be partitions of nonincreasing integers. 
Lemmas 2.3 2.5 enable to consider Problem 1 in the case when there do not exist $i \in\{1, \ldots, n\}$ and $j \in\{1, \ldots, m\}$ such that $c_{i}=d_{j}$. More precisely, we have the following result:

Proposition 2.6. Consider the partitions $\mathbf{a}, \mathbf{b}, \mathbf{c}$ and $\mathbf{d}$ as given in (2.29)(2.32.

Let $x$ be such that there exist $w \in\{1, \ldots, n\}$ and $u \in\{1, \ldots, m\}$ such that

$$
c_{w}=d_{u}=x .
$$

Let

$$
\mathbf{d}^{\prime}:=\left(d_{1}, \ldots, d_{u-1}, d_{u+1}, \ldots, d_{m}\right)
$$

and

$$
\mathbf{c}^{\prime}:=\left(c_{1}, \ldots, c_{w-1}, c_{w+1}, \ldots, c_{n}\right) \text {. }
$$

Then there exists a partition $\mathbf{g}=\left(g_{1}, \ldots, g_{m+s}\right)$ such that

$$
\begin{aligned}
& \mathrm{g} \prec^{\prime}(\mathbf{d}, \mathbf{a}) \\
& \mathrm{g} \prec^{\prime}(\mathbf{c}, \mathbf{b})
\end{aligned}
$$

if and only if there exists a partition $\mathbf{g}^{\prime}=\left(g_{1}^{\prime}, \ldots, g_{m+s-1}^{\prime}\right)$ such that

$$
\begin{gathered}
\mathbf{g}^{\prime} \prec^{\prime}\left(\mathbf{d}^{\prime}, \mathbf{a}\right) \\
\mathbf{g}^{\prime} \prec^{\prime}\left(\mathbf{c}^{\prime}, \mathbf{b}\right) .
\end{gathered}
$$

Proof. Let there exists $\mathrm{g}$ which satisfies (2.33) and (2.34). By Lemma 2.5, there exists a partition $\mathbf{g}^{\prime \prime}=\left(g_{1}^{\prime \prime}, \ldots, g_{m+s}^{\prime \prime}\right)$ such that there exists $l \in\{1, \ldots, m+s\}$ with $g_{l}^{\prime \prime}=x$, and such that

$$
\begin{aligned}
& \mathrm{g}^{\prime \prime} \prec^{\prime}(\mathbf{d}, \mathbf{a}) \\
& \mathrm{g}^{\prime \prime} \prec^{\prime}(\mathbf{c}, \mathbf{b}) .
\end{aligned}
$$

Now, let $\mathbf{g}^{\prime}=\mathbf{g}^{\prime \prime} \backslash\{x\}$.

By Lemma 2.3. we have that (2.35) and (2.36) are satisfied, as wanted.

Conversely, let $\mathbf{g}^{\prime}$ be such that (2.35) and (2.36) are satisfied. Let $\mathbf{g}:=\mathbf{g}^{\prime} \cup\{x\}$. Then by Lemma 2.3, (2.33) and (2.34) are valid, as wanted. $\mathrm{C}$

Thus, from now on without loss of generality, we shall consider Problem 1 only for partitions $\mathbf{c}$ and $\mathbf{d}$ such that for all $i \in\{1, \ldots, n\}$ and $j \in\{1, \ldots, m\}$, we have $c_{i} \neq d_{j}$. 
3. Sets $\mathbf{S}$ and $\boldsymbol{\Delta}$. Let $\mathbf{a}, \mathbf{b}, \mathbf{c}$ and $\mathbf{d}$ be partitions as given in (2.29)-(2.32).

Moreover, let $c_{1} \geq \cdots \geq c_{n}$ and $d_{1} \geq \cdots \geq d_{m}$ be such that there do not exist $i \in\{1, \ldots, n\}$ and $j \in\{1, \ldots, m\}$ such that $c_{i}=d_{j}$.

In this section, we shall define certain labels on the sequences $c_{1}, \ldots, c_{n}$ and $d_{1}, \ldots, d_{m}$. We are going to define the set $S$ of the indices of chosen integers among $c_{1} \geq \cdots \geq c_{n}$ and the set $\Delta$ as the indices of special ones among $d_{1} \geq \cdots \geq d_{m}$.

We note that the definition is similar to, but not the same as the one from Section 3.1. in [8]. It is given by recursion on $\left(c_{1}, \ldots, c_{n}\right) \cup\left(d_{1}, \ldots, d_{m}\right)$, starting from the smallest integer. More precisely:

Put $S:=\emptyset, \Delta:=\emptyset$.

The definitions are given recursively, so first we take the smallest integer from $\left(c_{1}, \ldots, c_{n}, d_{1}, \ldots, d_{m}\right)$. Then we have two possibilities $(a)$ and $(b)$, depending on whether the chosen integer belongs to $\left(d_{1}, \ldots, d_{m}\right)$ or it belongs to $\left(c_{1}, \ldots, c_{n}\right)$.

(a) If the observed integer is among $\left(d_{1}, \ldots, d_{m}\right)$, say $d_{j}$, then let

$$
q_{j}=s-\sharp\left\{c_{i}<d_{j} \mid i \in S\right\}+\sharp\{i>j \mid i \notin \Delta\}+1 .
$$

If $q_{j}>s$ or $q_{j}<0$, we add $j$ to $\Delta$, i.e., $\Delta:=\Delta \cup\{j\}$.

If $s \geq q_{j} \geq 0$, we check the following equation:

$$
\sum_{\substack{c_{i}<d_{j} \\ i \in S}} c_{i}<\sum_{\substack{i>j \\ i \notin \Delta}} d_{i}+d_{j}+\sum_{i=q_{j}+1}^{s} a_{i} .
$$

If $d_{j}$ satisfies (3.2), then we add $j$ to $\Delta$, i.e., $\Delta:=\Delta \cup\{j\}$.

(b) If the observed integer is among $\left(c_{1}, \ldots, c_{n}\right)$, say $c_{j}$, then let

$$
q_{j}^{\prime}=k-\sharp\left\{d_{i}<c_{j} \mid i \in \Delta\right\}+\sharp\{i>j \mid i \notin S\}+1 .
$$

If $q_{j}^{\prime}>k$ or $q_{j}^{\prime}<0$, we add $j$ to the set $S$, i.e., $S:=S \cup\{j\}$.

If $k \geq q_{j}^{\prime} \geq 0$, we check the following inequality:

$$
\sum_{\substack{d_{i}<c_{j} \\ i \in \Delta}} d_{i}<\sum_{\substack{i>j \\ i \notin S}} c_{i}+c_{j}+\sum_{i=q_{j}^{\prime}+1}^{k} b_{i} .
$$

If (3.4) is satisfied, then we add $j$ to the set $S$, i.e., $S:=S \cup\{j\}$. 
Now, we select the next smallest integer among $\left(c_{1}, \ldots, c_{n}, d_{1}, \ldots, d_{m}\right)$, and proceed.

In this way, we have defined the sets $\Delta$ and $S$. These sets will play an essential role in the rest of the paper. Also, it is trivial to see that these definitions are symmetric, i.e., one can be obtained from the another by simply exchanging $\Delta, d_{i}, a_{i}$ and $q_{i}$ with $S, c_{i}, b_{i}$ and $q_{i}^{\prime}$, respectively, and vice-versa.

3.1. Some additional notation. We also introduce a couple of definitions in order to simplify and clarify further notation.

Let $h:=\sharp S$. We shall denote by $c^{1} \geq \cdots \geq c^{h}$ the nonincreasing ordering of $c_{i}$ 's such that $i \in S$. Also, for each $c^{x}, x=1, \ldots, h$, we shall define

$$
z_{x}:=\max \left\{i \mid d_{i}>c^{x}\right\}
$$

and

$$
m_{x}:=\max \left\{i \mid a_{i}>c^{x}\right\}
$$

i.e.,

$$
d_{z_{x}}>c^{x}>d_{z_{x}+1}, \quad a_{m_{x}}>c^{x} \geq a_{m_{x}+1} .
$$

We also set $c^{0}:=c_{0}, c^{h+1}:=c_{n+1}, z_{0}:=0, z_{h+1}:=m, m_{0}:=0$ and $m_{h+1}:=s$.

Moreover, we define

$$
t_{x}:=s-(h-x)+\sharp\left\{i \notin \Delta \mid d_{i}<c^{x}\right\}, \quad x=0, \ldots, h,
$$

and $t_{h+1}:=s+1$.

Analogously, let $h^{\prime}:=\sharp \Delta$. We shall denote by $d^{1} \geq \cdots \geq d^{h^{\prime}}$ the nonincreasing ordering of $d_{i}$ 's such that $i \in \Delta$. Also, for each $d^{x}, x=1, \ldots, h^{\prime}$, we shall define

$$
z_{x}^{\prime}:=\max \left\{i \mid c_{i}>d^{x}\right\}
$$

and

$$
m_{x}^{\prime}:=\max \left\{i \mid b_{i}>d^{x}\right\}
$$

i.e.,

$$
c_{z_{x}^{\prime}}>d^{x}>c_{z_{x}^{\prime}+1}, \quad b_{m_{x}^{\prime}}>d^{x} \geq b_{m_{x}^{\prime}+1} .
$$

We also set $d^{0}:=d_{0}, d^{h^{\prime}+1}:=d_{m+1}, z_{0}^{\prime}:=0, z_{h^{\prime}+1}^{\prime}:=n, m_{0}^{\prime}:=0$ and $m_{h^{\prime}+1}^{\prime}:=k$.

Moreover, we define

$$
t_{x}^{\prime}:=k-\left(h^{\prime}-x\right)+\sharp\left\{i \notin S \mid c_{i}<d^{x}\right\}, \quad x=0, \ldots, h^{\prime},
$$

and $t_{h^{\prime}+1}^{\prime}:=k+1$. 
4. Properties of the sets $\mathbf{S}$ and $\boldsymbol{\Delta}$. In this section, we present some auxiliary lemmas. Many of them are analogous to lemmas from Section 3.1 in [8]. However, since the definition of the sets $S$ and $\Delta\left(D^{C}\right)$ are different from the analogous definition given in [8], we have to re-state and re-prove all of the lemmas given in [8].

LEMmA 4.1. Let $j \in\{1, \ldots, m\}$ and $x \in\{0, \ldots, h\}$ be such that $c^{x}>d_{j}>c^{x+1}$, and $j \in \Delta$. Then

$$
z_{x}+1, z_{x}+2, \ldots, j-1 \in \Delta
$$

Proof. If $x=h$, then $q_{j}>s$. Moreover, by the definition of $q_{i}$ 's we have that $q_{z_{x}+1} \geq \cdots \geq q_{j-1} \geq q_{j}>s$, thus proving (4.1).

If $x<h$, then $d_{j}>c^{h}$. Suppose on the contrary to (4.1) that among $\left\{z_{x}+\right.$ $1, \ldots, j-1\}$ there are indices that are not from $\Delta$. Denote by $u$ the largest of them. Then since $q_{j}=q_{u}$ (by the definition), we have that $s \geq q_{j}=q_{u} \geq 0$, and thus $d_{j}$ satisfies (3.2), i.e.,

$$
\sum_{i=x+1}^{h} c^{i}<d_{j}+\sum_{\substack{i>j \\ i \notin \Delta}} d_{i}+\sum_{i=q_{j}+1}^{s} a_{i}
$$

while $d_{u}$ does not satisfy $(\underline{3.2}$, and so we have

$$
\sum_{i=x+1}^{h} c^{i} \geq d_{u}+\sum_{\substack{i>j \\ i \notin \Delta}} d_{i}+\sum_{i=q_{j}+1}^{s} a_{i}
$$

Last two equations together give that $d_{j}>d_{u}$, which is a contradiction. This proves (4.1), as wanted.

Now, as a corollary of Lemma 4.1. we have:

Lemma 4.2. Let $c^{x}>d_{j}>c^{x+1}$, and let $j \notin \Delta$. Then $j+1, \ldots, z_{x+1} \notin \Delta$.

Denote by $w_{x}$ the number of $d_{i}$ 's such that $i \notin \Delta$ and such that $c^{x}>d_{i}>c^{x+1}$. Then from the definition of $z_{x}$ and $w_{x}$, by Lemmas 4.1 and 4.2, we have

$$
d_{z_{x}}>c^{x}>\underbrace{d_{z_{x}+1} \geq d_{z_{x}+2} \geq \ldots \geq d_{z_{x+1}-w_{x}}}_{\in \Delta} \geq \underbrace{d_{z_{x+1}-w_{x}+1} \geq \ldots \geq d_{z_{x+1}}}_{\notin \Delta}>c^{x+1} .
$$

In particular, we have

$$
t_{x+1}=t_{x}-w_{x}+1
$$


Completely analogously, we obtain the following dual results:

LEMmA 4.3. Let $j \in\{1, \ldots, n\}$ and $x \in\left\{0, \ldots, h^{\prime}\right\}$ be such that $d^{x}>c_{j}>d^{x+1}$, and $j \in S$. Then

$$
z_{x}^{\prime}+1, z_{x}^{\prime}+2, \ldots, j-1 \in S
$$

Lemma 4.4. Let $d^{x}>c_{j}>d^{x+1}$, and let $j \notin S$. Then $j+1, \ldots, z_{x+1}^{\prime} \notin S$.

Denote by $w_{x}^{\prime}$ the number of $c_{i}$ 's such that $i \notin S$ and such that $d^{x}>c_{i}>d^{x+1}$. Then from the definition of $z_{x}^{\prime}$ and $w_{x}^{\prime}$, by Lemmas 4.3 and 4.4 , we have

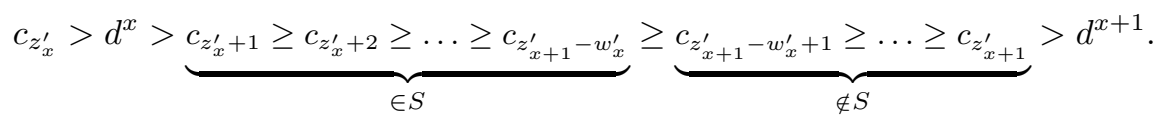

The proofs of the following two lemmas follow directly by the definitions of $z_{i}, z_{i}^{\prime}$, $t_{i}$ and $t_{i}^{\prime}$ :

Lemma 4.5. Let $j \in S$. Let $i \in\{1, \ldots, h\}$ be such that $c_{j}=c^{i}$ and let $x \in$ $\left\{0, \ldots, h^{\prime}\right\}$ be such that $d^{x}>c_{j}>d^{x+1}$. Then

$$
z_{i}+t_{i}=j+t_{x}^{\prime}
$$

Lemma 4.6. Let $j \in \Delta$. Let $i \in\left\{1, \ldots, h^{\prime}\right\}$ be such that $d_{j}=d^{i}$ and let $x \in\{0, \ldots, h\}$ be such that $c^{x}>d_{j}>c^{x+1}$. Then

$$
z_{i}^{\prime}+t_{i}^{\prime}=j+t_{x}
$$

Lemma 4.7. Let $x \in\{1, \ldots, h\}$, and let $m_{x} \geq t_{x}$. Let

$$
\sum_{i=z_{u}+t_{u}}^{z_{u}+m_{u}} e_{i} \leq \sum_{i=u}^{h} c^{i}-\sum_{\substack{i>z_{u} \\ i \notin \Delta}} d_{i}-\sum_{i=m_{u}+1}^{s} a_{i}
$$

for all $u \geq x$ such that $m_{u} \geq t_{u}$.

Here $\left(e_{z_{u}+t_{u}}, \ldots, e_{z_{u}+m_{u}}\right)$ are defined as the smallest $m_{u}-t_{u}+1$ elements among $\left(a_{1}, \ldots, a_{m_{u}}, d_{1}, \ldots, d_{z_{u}}\right)$.

$$
\text { Let } d_{j} \in\left(e_{z_{x}+t_{x}}, \ldots, e_{z_{x}+m_{x}}\right) \text {. Then } j \notin \Delta \text {. }
$$


Proof. The proof goes by induction on $x$. Let $x=h$. Then $m_{h} \geq s\left(=t_{h}\right)$. Hence, $a_{s}>c^{h}$. By (4.4) for $u=h$, we have that

$$
c^{h}<\min \left(d_{z_{h}}, a_{s}\right)=e_{z_{h}+s} \leq c^{h}
$$

which is a contradiction. Hence, the condition $m_{x} \geq t_{x}$ implies that $x<h$. Thus, let $x<h$ and suppose that our claim is valid for all $i>x$, for which $m_{i} \geq t_{i}$.

If $m_{x}=t_{x}$, then $e_{z_{x}+t_{x}}=d_{j}$ and $j=z_{x}$. Thus $s \geq q_{j}=m_{x} \geq 0$. Moreover, by (4.4), $d_{j}$ satisfies

$$
d_{j}=d_{z_{x}} \leq \sum_{i=x}^{h} c^{i}-\sum_{\substack{i>j \\ i \notin \Delta}} d_{i}-\sum_{i=m_{x}+1}^{s} a_{i}
$$

i.e., by (3.2) $j \notin \Delta$, as wanted.

Now, consider the case $m_{x}>t_{x}$. First we shall prove that then $m_{x+1} \geq t_{x+1}$. Suppose on the contrary that $m_{x+1}<t_{x+1}$. Then $t_{x}<m_{x} \leq m_{x+1}<t_{x+1}$, i.e.,

$$
t_{x}+2 \leq t_{x+1}
$$

However, by the definition of $t_{x}, t_{x+1}$ and $w_{x}$ we have that $t_{x}=t_{x+1}+w_{x}-1 \geq t_{x+1}-1$. Thus, (4.5) is impossible.

Moreover, we shall prove that $m_{x}>t_{x}$ implies $z_{x}=z_{x+1}-w_{x}$, i.e., that all $d_{i}$ 's such that $c^{x}>d_{i}>c^{x+1}$, satisfy $i \notin \Delta$.

In order to prove this, suppose on the contrary that $z_{x}<z_{x+1}-w_{x}$. Then, $z_{x+1}-w_{x} \in \Delta$ and $d_{z_{x+1}-w_{x}}<c^{x}$ (see (4.2)). By the induction hypothesis, we have that $d_{z_{x+1}-w_{x}}$ is not among $\left(e_{z_{x+1}+t_{x+1}}, \ldots, e_{z_{x+1}+m_{x+1}}\right)$, i.e., all $e_{i}$ 's for $i=$ $z_{x+1}+t_{x+1}, \ldots, z_{x+1}+m_{x+1}$ are less than or equal to $d_{z_{x+1}-w_{x}}$. This implies that among $\left(e_{z_{x+1}+t_{x+1}}, \ldots, e_{z_{x+1}+m_{x+1}}\right)$, there are at most $w_{x} d_{i}$ 's, and at least $m_{x+1}-$ $t_{x+1}+1-w_{x}=m_{x+1}-t_{x} a_{i}$ 's. Thus $a_{t_{x}+1}$ is among $\left(e_{z_{x+1}+t_{x+1}}, \ldots, e_{z_{x+1}+m_{x+1}}\right)$ and so $a_{t_{x}+1} \leq d_{z_{x+1}-w_{x}}<c^{x}$. This means that $m_{x} \leq t_{x}$, which contradicts $m_{x}>t_{x}$.

Hence, $z_{x}=z_{x+1}-w_{x}$, and so $z_{x}+t_{x}+1=z_{x+1}+t_{x+1}$.

Now we have that $d_{j}$ is among $\left(e_{z_{x}+t_{x}+1}, \ldots, e_{z_{x}+m_{x}}\right)$ or $d_{j}$ is $e_{z_{x}+t_{x}}$.

If $d_{j}$ is among $\left(e_{z_{x}+t_{x}+1}, \ldots, e_{z_{x}+m_{x}}\right)$, then since $z_{x}+t_{x}+1=z_{x+1}+t_{x+1}, d_{j}$ is among $\left(e_{z_{x+1}+t_{x+1}}, \ldots, e_{z_{x+1}+m_{x+1}}\right)$. Hence, by the induction hypothesis we have that $j \notin \Delta$. 
If $d_{j}$ is $e_{z_{x}+t_{x}}$, then (4.4) gives

$$
d_{j}+\sum_{i=z_{x}+t_{x}+1}^{z_{x}+m_{x}} e_{i}=\sum_{i=z_{x}+t_{x}}^{z_{x}+m_{x}} e_{i} \leq \sum_{i=x}^{h} c^{i}-\sum_{\substack{i>z_{x} \\ i \notin \Delta}} d_{i}-\sum_{i=m_{x}+1}^{s} a_{i}
$$

The sequence $\left(e_{z_{x}+t_{x}+1}, \ldots, e_{z_{x}+m_{x}}\right)$ consists of $d_{j+1}, \ldots, d_{z_{x}}$ and $a_{q_{j}+1}, \ldots, a_{m_{x}}$. Hence,

$$
q_{j} \leq m_{x} \leq s
$$

By the induction hypothesis $j+1, \ldots, z_{x} \notin \Delta$. Thus, (4.6) gives

$$
d_{j} \leq \sum_{i=x}^{h} c^{i}-\sum_{\substack{i>j \\ i \notin \Delta}} d_{i}-\sum_{i=q_{j}+1}^{s} a_{i}
$$

Last implies that $q_{j} \geq 0$, which together with (4.7), (4.8) and (3.2), gives that $j \notin \Delta$, as wanted. $\square$

Completely analogously, we have the dual result:

LEMMA 4.8. Let $y \in\left\{1, \ldots, h^{\prime}\right\}$, and let $m_{y}^{\prime} \geq t_{y}^{\prime}$. Let

$$
\sum_{i=z_{u}^{\prime}+t_{u}^{\prime}}^{z_{u}^{\prime}+m_{u}^{\prime}} e_{i}^{\prime} \leq \sum_{i=u}^{h^{\prime}} d^{i}-\sum_{\substack{i>z_{u}^{\prime} \\ i \notin S}} c_{i}-\sum_{i=m_{u}^{\prime}+1}^{k} b_{i}
$$

for all $u \geq y$ such that $m_{u}^{\prime} \geq t_{u}^{\prime}$. Here, $\left(e_{z_{u}^{\prime}+t_{u}^{\prime}}^{\prime}, \ldots, e_{z_{u}^{\prime}+m_{u}^{\prime}}^{\prime}\right)$ are defined as the smallest $m_{u}^{\prime}-t_{u}^{\prime}+1$ elements among $\left(b_{1}, \ldots, b_{m_{u}^{\prime}}, c_{1}, \ldots, c_{z_{u}^{\prime}}\right)$.

Let $c_{j} \in\left(e_{z_{y}^{\prime}+t_{y}^{\prime}}^{\prime}, \ldots, e_{z_{y}^{\prime}+m_{y}^{\prime}}^{\prime}\right)$. Then $j \notin S$.

Lemma 4.9. For every $x=0, \ldots, h-1$, we have $z_{x+1}+t_{x+1}>z_{x}+t_{x}$.

Proof. From the definition of $w_{x}$ we have $w_{x} \leq z_{x+1}-z_{x}$, and so

$$
z_{x+1}+t_{x+1}=z_{x+1}+t_{x}+1-w_{x} \geq z_{x}+t_{x}+1>z_{x}+t_{x}
$$

as wanted.

Analogously, we have:

LEMma 4.10. For every $x=0, \ldots, h^{\prime}-1$, we have $z_{x+1}^{\prime}+t_{x+1}^{\prime}>z_{x}^{\prime}+t_{x}^{\prime}$. 
From (4.2) and the definitions of $q_{j}$ 's and $t_{i}$ 's (see (3.1) and (3.5)), we have the following result:

Lemma 4.11. Let $x \in\{0, \ldots, h\}$. Then

$$
q_{z_{x}+1}=q_{z_{x}+2}=\cdots=q_{z_{x+1}-w_{x}}=t_{x}+1
$$

and

$$
q_{z_{x+1}-w_{x}+j}=t_{x}-j+1, \quad j=1, \ldots, w_{x} .
$$

In particular, if $w_{x}>0$ (i.e., if $t_{x+1} \leq t_{x}$ ), then for every $i$ such that $t_{x+1} \leq i \leq t_{x}$ there exists $j \notin \Delta$ such that $c^{x}>d_{j}>c^{x+1}$ and $q_{j}=i$.

From (4.3) and the definitions of $q_{j}^{\prime}$ 's and $t_{i}^{\prime}$ 's (see (3.3) and (3.6)), we have the following result:

Lemma 4.12. Let $x \in\left\{0, \ldots, h^{\prime}\right\}$. Then,

$$
q_{z_{x}^{\prime}+1}^{\prime}=q_{z_{x}^{\prime}+2}^{\prime}=\cdots=q_{z_{x+1}^{\prime}-w_{x}^{\prime}}^{\prime}=t_{x}^{\prime}+1
$$

and

$$
q_{z_{x+1}^{\prime}-w_{x}^{\prime}+j}^{\prime}=t_{x}^{\prime}-j+1, \quad j=1, \ldots, w_{x}^{\prime}
$$

In particular, if $w_{x}^{\prime}>0$ (i.e., if $t_{x+1}^{\prime} \leq t_{x}^{\prime}$ ), then for every $i$ such that $t_{x+1}^{\prime} \leq i \leq t_{x}^{\prime}$ there exists $j \notin S$ such that $d^{x}>c_{j}>d^{x+1}$ and $q_{j}^{\prime}=i$.

Lemma 4.13. Let $x=0, \ldots, h$. Suppose that the condition (4.4) is valid for all $u=x+1, \ldots, h$ such that $m_{u} \geq t_{u}$. Then

$$
t_{x} \geq 0
$$

Proof. The proof goes by induction on $x$.

For $x=h$, we have $t_{h}=s \geq 0$.

Now, let $x<h$. By the induction hypothesis we have that $t_{x+1} \geq 0$.

By the definition of $t_{i}$ 's we have

$$
t_{x}=t_{x+1}-1+w_{x}
$$

Hence, if $t_{x+1}>0$ or $w_{x}>0$, we directly obtain $t_{x} \geq 0$, as wanted. So, the only remaining case is if $t_{x+1}=0$ and $w_{x}=0$ (and hence, $t_{x}=-1$ ). The set 
$\left\{e_{z_{x+1}}, \ldots, e_{z_{x+1}+m_{x+1}}\right\}$ has $m_{x+1}+1$ elements, and since it consists of $d_{i}$ 's and $a_{i}$ 's strictly greater than $c^{x+1}$, there must be at least one $d_{j}$ among them (since $\left.a_{m_{x+1}}>c^{x+1} \geq a_{m_{x+1}+1}\right)$. Then $d_{z_{x+1}} \in\left\{e_{z_{x+1}}, \ldots, e_{z_{x+1}+m_{x+1}}\right\}$. So, by Lemma 4.7 we have that $z_{x+1} \notin \Delta$. By the definition of $\Delta$ this gives that $q_{z_{x+1}} \geq 0$. Let $v \geq 0$ be such that $c^{x-v-1}>d_{z_{x+1}}>c^{x-v}$. Then by the definition of $q_{i}$ 's and $t_{j}$ 's we have that $q_{z_{x+1}}=t_{x}-v$, and thus $t_{x} \geq v \geq 0$. Last contradicts our assumption that $t_{x}=-1$. $\square$

Analogously, we obtain the dual result:

Lemma 4.14. Let $y=0, \ldots, h^{\prime}$. Suppose that the condition 4.9) is valid for all $u=y+1, \ldots, h^{\prime}$ such that $m_{u}^{\prime} \geq t_{u}^{\prime}$. Then

$$
t_{y}^{\prime} \geq 0
$$

LEMMA 4.15. For every $j$ such that $t_{0}<j \leq s$, there exists $i \in\{1, \ldots, h\}$, such that $t_{i}=j$.

Proof. Suppose that for some $j$ with $t_{0}<j \leq s$, there are no $i \in\{1, \ldots, h\}$, such that $t_{i}=j$. Then from $t_{i+1} \leq t_{i}+1$, for $i=0, \ldots, h-1$, we have that $t_{i}<j$ implies $t_{i+1}<j$, for every $i=0, \ldots, h-1$. Since $t_{0}<j$, this would imply that $t_{h}<j$, which is a contradiction since $t_{h}=s$.

Analogously, we have the dual result:

Lemma 4.16. For every $j$ such that $t_{0}^{\prime}<j \leq k$, there exists $i \in\left\{1, \ldots, h^{\prime}\right\}$, such that $t_{i}^{\prime}=j$.

LEMMA 4.17. [8, Lemma 4.9] Let $u_{1} \geq \cdots \geq u_{k}$ and $v_{1} \geq \cdots \geq v_{k}$ be integers. If

$$
\sharp\left\{i \mid u_{i}>v_{j}\right\} \geq j, \quad \text { for all } \quad j=1, \ldots, k,
$$

then

$$
\sum_{i=1}^{k} u_{i} \geq \sum_{i=1}^{k} v_{i}+k
$$

LEMMA 4.18. For every $i=0, \ldots, h-1$, we have $t_{i}<s$, while $t_{h}=s$.

Proof. By the definition of $t_{h}$ we directly obtain that $t_{h}=s$. Now, suppose that there exists $i \in\{0, \ldots, h-1\}$ such that $t_{i} \geq s$. Let $y:=\max \left\{i \mid t_{i} \geq s\right\}$. Note that

$$
t_{h-1}=s-1 .
$$


Indeed, by the definition $t_{h-1}=s-1+w_{h-1}$. However, if $w_{h-1}>0$, then $c^{h-1}>d_{z_{h}}$, with $z_{h} \notin \Delta$, and $q_{z_{h}}=s$. Hence, by (3.2) we would have $c^{h} \geq d_{z_{h}}$, which is impossible. Thus (4.10) is satisfied.

So $y<h-1$. Thus, $t_{y} \geq s>t_{y+1}$ and so $w_{y} \geq 2$. By Lemma 4.11 there exists $f \notin \Delta$ such that $c^{y}>d_{f}>c^{y+1}$ and $q_{f}=s$. Hence, $d_{f}$ does not satisfy (3.2), i.e.,

$$
\sum_{i=y+1}^{h} c^{i} \geq \sum_{\substack{i \geq f \\ i \notin \Delta}} d_{i}
$$

We note that the number of summands on both sides of (4.11) is equal.

For every $j=y+1, \ldots, h$, we have $t_{j} \leq s=q_{f}$. From the definitions of $q_{i}$ 's (3.1) and $t_{i}$ 's (3.5), we obtain $\sharp\left\{i \notin \Delta \mid i \geq f, d_{i}>c^{j}\right\}=\sharp\left\{i \notin \Delta \mid f \leq i \leq z_{j}\right\} \geq j-y$, $j=y+1, \ldots, h$. This together with Lemma 4.17 contradicts (4.11). Thus, $t_{i}<s$, for all $i=0, \ldots, h-1$, as wanted.

And analogously, we have the dual result:

Lemma 4.19. For every $i=0, \ldots, h^{\prime}-1$, we have $t_{i}^{\prime}<k$, while $t_{h^{\prime}}^{\prime}=k$.

5. Main result. Consider partitions $\mathbf{a}, \mathbf{b}, \mathbf{c}$ and $\mathbf{d}$ as given in (2.29)-(2.32)

In this section, we give a complete and explicit solution to Problem 1

As it was proven in Subsection [2.2, it is enough to resolve the Problem 1 in the case when partitions $\mathbf{c}$ and $\mathbf{d}$ do not have same elements.

Thus we assume that $\mathbf{c}$ and $\mathbf{d}$ are such that there are no $i \in\{1, \ldots, n\}$ and no $j \in\{1, \ldots, m\}$ such that $c_{i}=d_{j}$. For such $\mathbf{a}, \mathbf{b}, \mathbf{c}$ and $\mathbf{d}$ we define the sets $S$ and $\Delta$, together with labels $t_{x}, t_{y}^{\prime}, c^{x}$ and $d^{y}$ as in Section 3 .

The solution of Problem 1 is given in the following theorem:

THEOREM 5.1. Let $m, n, k$ and $s$ be nonnegative integers such that $m+s=n+k$. Let $\mathbf{a}, \mathbf{b}$, $\mathbf{c}$ and $\mathbf{d}$ be partitions as given in (2.29)- (2.32).

Then there exists a partition $\mathbf{g}=\left(g_{1}, \ldots, g_{m+s}\right)$, such that

$$
\begin{aligned}
& \mathrm{g} \prec^{\prime}(\mathbf{d}, \mathbf{a}) \\
& \mathrm{g} \prec^{\prime}(\mathbf{c}, \mathbf{b})
\end{aligned}
$$

if and only if the following conditions are valid:

$$
\text { (i) } \sum_{i=1}^{n} c_{i}+\sum_{i=1}^{k} b_{i}=\sum_{i=1}^{m} d_{i}+\sum_{i=1}^{s} a_{i},
$$




$$
\begin{aligned}
& \text { (ii) } \sum_{i=x+1}^{h} c^{i} \geq \sum_{\substack{d_{i}<c^{x} \\
i \notin \Delta}} d_{i}+\sum_{i=t_{x}+1}^{s} a_{i}, \quad x=0, \ldots, h, \\
& \text { (iii) } \sum_{i=y+1}^{h^{\prime}} d^{i} \geq \sum_{\substack{c_{i}<d^{y} \\
i \notin S}} c_{i}+\sum_{i=t_{y}^{\prime}+1}^{k} b_{i}, \quad y=0, \ldots, h^{\prime} .
\end{aligned}
$$

5.1. Auxiliary results. In this section, we give four crucial lemmas for the proof of the necessity part of Theorem 5.1 .

Lemma 5.2. Let $\mathbf{a}, \mathbf{b}, \mathbf{c}$ and $\mathbf{d}$ be partitions as given in (2.29)-2.32). Let $\mathbf{g}$ be a partition such that

$$
\mathbf{g} \prec^{\prime}(\mathbf{d}, \mathbf{a}) .
$$

Let $d_{j}, j \in\{1, \ldots, m\}$ be such that $j \in \Delta$.

Let $x \in\{0, \ldots, h\}$ be such that

$$
c^{x}>d_{j}>c^{x+1} .
$$

Suppose that

$$
\sum_{i=y+1}^{h} c^{i} \geq \sum_{\substack{d_{i}<c^{y} \\ i \notin \Delta}} d_{i}+\sum_{i=t_{y}+1}^{s} a_{i}, \quad \text { for } \quad y \geq x
$$

and that for all $d_{i}<c^{x+1}, i \in \Delta$, the following holds

$$
d_{i} \geq g_{i+t_{w}}, \quad \text { where } w \text { is such that } \quad c^{w}>d_{i}>c^{w+1} .
$$

Then

$$
d_{j} \geq g_{j+t_{x}}
$$

Proof. The proof is split into two parts depending on the value of $x$. 
Let $x=h$. By (3.5), we have that $t_{h}=s$, hence (5.7) follows from (5.3).

Let $x<h$. Since $c^{x}>d_{j}>c^{x+1}$ and $j \in \Delta$, we have that $w_{x}<z_{x+1}-z_{x}$.

Let $h_{u}:=\min \left\{i \mid d_{i-u+1}<g_{i}\right\}, u=1, \ldots, s\left(h_{0}:=0, h_{s+1}:=m+s+1\right)$.

If $h_{t_{x}+1} \geq z_{x+1}+t_{x+1}$, then for all $i<z_{x+1}+t_{x+1}-t_{x}=z_{x+1}-w_{x}+1$, we have $d_{i} \geq g_{i+t_{x}}$, as wanted. Thus it is sufficient to prove that $h_{t_{x}+1} \geq z_{x+1}+t_{x+1}$.

Suppose on the contrary that $h_{t_{x}+1} \leq z_{x+1}+t_{x+1}-1$, and let $u$ be such that $h_{u} \leq z_{x+1}+t_{x+1}-1<h_{u+1}$. Then $u \geq t_{x}+1$. From (5.3) by Lemma 2.2. we have that the following is valid:

$$
\sum_{i=z_{x+1}+t_{x+1}}^{m+s} g_{i} \geq \sum_{i=z_{x+1}+t_{x+1}-u}^{m} d_{i}+\sum_{i=u+1}^{s} a_{i} .
$$

Moreover, since $q_{z_{x+1}-w_{x}}=t_{x}+1$ and since (5.5) is valid, by Lemmas 4.13 and 4.18, we have $s \geq q_{z_{x+1}-w_{x}} \geq 0$. Hence $z_{x+1}-w_{x} \in \Delta$ implies that (3.2) is valid for $d_{z_{x+1}-w_{x}}$, i.e.,

$$
\sum_{i=x+1}^{h} c^{i}<\sum_{\substack{i>z_{x+1}-w_{x} \\ i \notin \Delta}} d_{i}+d_{z_{x+1}-w_{x}}+\sum_{i=q_{z_{x+1}-w_{x}}+1}^{s} a_{i} .
$$

By (5.4) and (5.6), we have

$$
\sum_{i=z_{x+1}+t_{x+1}}^{m+s} g_{i} \leq \sum_{i=x+1}^{h} c^{i}+\sum_{i=z_{x+1}+1}^{m} d_{i}-\sum_{\substack{i>z_{x+1} \\ i \notin \Delta}} d_{i}
$$

Inequalities (5.9) and (5.10) together give

$$
\sum_{i=z_{x+1}+t_{x+1}}^{m+s} g_{i}<\sum_{i=z_{x+1}-w_{x}}^{m} d_{i}+\sum_{i=t_{x}+2}^{s} a_{i} .
$$

Since $w_{x}=t_{x}-t_{x+1}+1$ and $u \geq t_{x}+1$, (5.8) and (5.11) together give

$$
\sum_{i=z_{x+1}+t_{x+1}-u}^{z_{x+1}-t_{x}+t_{x+1}-2} d_{i}<\sum_{i=t_{x}+2}^{u} a_{i}
$$

Note that the number of the summands on both sides of (5.12) is equal. We shall prove that the smallest summand on the left hand side, $d_{z_{x+1}-t_{x}+t_{x+1}-2}$, is larger than or equal to the largest summand on the right hand side, $a_{t_{x}+2}$, of (5.12), thus obtaining the contradiction. 
Equation (5.9), together with (5.5) for $y=x$, gives $d_{z_{x+1}-w_{x}}>a_{t_{x}+1}$, which finishes our proof. $\square$

Now we can state the dual result:

Lemma 5.3. Let $\mathbf{a}, \mathbf{b}, \mathbf{c}$ and $\mathbf{d}$ be partitions as given in (2.29)-2.32). Let $\mathbf{g}$ be a partition such that

$$
\mathbf{g} \prec^{\prime}(\mathbf{c}, \mathbf{b}) \text {. }
$$

Let $c_{j}, j \in\{1, \ldots, n\}$ be such that $j \in S$.

Let $y \in\left\{0, \ldots, h^{\prime}\right\}$ be such that

$$
d^{y}>c_{j}>d^{y+1} .
$$

Suppose that

$$
\begin{gathered}
d^{i} \geq g_{z_{i}^{\prime}+t_{i}^{\prime}}, \quad i \geq y+1, \\
\sum_{i=x+1}^{h^{\prime}} d^{i} \geq \sum_{\substack{c_{i}<d^{x} \\
i \notin S}} c_{i}+\sum_{i=t_{x}^{\prime}+1}^{k} b_{i}, \quad \text { for } \quad x \geq y,
\end{gathered}
$$

and that for all $c_{i}<d^{y+1}, i \in S$, the following holds

$$
c_{i} \geq g_{i+t_{w}^{\prime}}, \quad \text { where } w \text { is such that } \quad d^{w}>c_{i}>d^{w+1} .
$$

Then

$$
c_{j} \geq g_{j+t_{y}^{\prime}}
$$

Lemma 5.4. Let $\mathbf{a}, \mathbf{b}, \mathbf{c}$ and $\mathbf{d}$ be partitions as given in (2.29)-2.32). Let $\mathbf{g}$ be a partition such that

$$
\mathbf{g} \prec^{\prime}(\mathbf{d}, \mathbf{a}) .
$$

Let $x \in\{0, \ldots, h-1\}$. Suppose that

$$
c^{i} \geq g_{z_{i}+t_{i}}, \quad i \geq x+1,
$$




$$
\sum_{i=y+1}^{h} c^{i} \geq \sum_{\substack{d_{i}<c^{y} \\ i \notin \Delta}} d_{i}+\sum_{i=t_{y}+1}^{s} a_{i}, \quad \text { for } \quad y \geq x+1
$$

and that for all $d_{i}<c^{x+1}, i \in \Delta$, the following holds

$$
d_{i} \geq g_{i+t_{w}}, \quad \text { where } w \text { is such that } \quad c^{w}>d_{i}>c^{w+1} .
$$

Then

$$
\sum_{i=x+1}^{h} c^{i} \geq \sum_{\substack{d_{i}<c^{x} \\ i \notin \Delta}} d_{i}+\sum_{i=t_{x}+1}^{s} a_{i}
$$

Proof. The proof is split into two parts. First let suppose that $h=x+1$. Then (5.17) becomes

$$
c^{h} \geq a_{s}
$$

Let $h_{u}:=\min \left\{i \mid d_{i-u+1}<g_{i}\right\}, u=1, \ldots, s\left(h_{0}:=0, h_{s+1}:=m+s+1\right)$. Let $j \in\{0, \ldots, s\}$ be such that

$$
h_{j}<z_{h}+t_{h}=z_{h}+s \leq h_{j+1} .
$$

Then (5.13) together with Lemma 2.2 gives

$$
\sum_{i=z_{h}+s}^{m+s} g_{i} \geq \sum_{i=z_{h}+s-j}^{m} d_{i}+\sum_{i=j+1}^{s} a_{i}
$$

Also, (5.14) and (5.16) give

$$
c^{h}+\sum_{i=z_{h}+1}^{m} d_{i} \geq \sum_{i=z_{h}+s}^{m+s} g_{i}
$$

Inequalities (5.19) and (5.20) give

$$
c^{h}+\sum_{i=z_{h}+1}^{m} d_{i} \geq \sum_{i=z_{h}+s-j}^{m} d_{i}+\sum_{i=j+1}^{s} a_{i} .
$$


If $j=s$, equation (5.21) becomes $c^{h} \geq d_{z_{h}}$, which is impossible by the definition of $z_{h}$. Thus, we have $j<s$ and then (5.21) becomes

$$
c^{h}+\sum_{i=z_{h}+1}^{z_{h}+s-j-1} d_{i} \geq \sum_{i=j+1}^{s} a_{i} .
$$

The last implies

$$
(s-j) c^{h} \geq c^{h}+\sum_{z_{h}+1}^{z_{h}+s-j-1} d_{i} \geq \sum_{i=j+1}^{s} a_{i} \geq(s-j) a_{s},
$$

i.e., we obtain (5.18), as wanted.

Now suppose that $x+1<h$.

If $w_{x}>0$, then by the definition of $z_{x+1}, w_{x}$ and by Lemmas 4.1 and 4.2, we have that $z_{x+1}-w_{x}+1 \notin \Delta$. Hence, $d_{z_{x+1}-w_{x}+1}$ does not satisfy (3.2), i.e., we have

$$
\sum_{i=x+1}^{h} c^{i} \geq d_{z_{x+1}-w_{x}+1}+\sum_{\substack{i>z_{x+1}-w_{x}+1 \\ i \notin \Delta}} d_{i}+\sum_{i=q_{z_{x+1}-w_{x}+1}+1}^{s} a_{i} .
$$

Moreover, we have

$$
d_{z_{x+1}-w_{x}+1}+\sum_{\substack{i>z_{x+1}-w_{x}+1 \\ i \notin \Delta}} d_{i}=\sum_{\substack{i>z_{x} \\ i \notin \Delta}} d_{i}
$$

and

$$
q_{z_{x+1}-w_{x}+1}=t_{x}
$$

Hence, (5.22) implies (5.17), as wanted.

Thus, we are left with the case $w_{x}=0$. Then

$$
\sum_{\substack{i>z_{x+1} \\ i \notin \Delta}}^{t_{x+1}=t_{x}+1} d_{i}=\sum_{\substack{i>z_{x} \\ i \notin \Delta}} d_{i} .
$$

We shall consider two subcases. Let $m_{x+1}<t_{x+1}$. Then

$$
c^{x+1} \geq a_{m_{x+1}+1} \geq a_{t_{x+1}}=a_{t_{x}+1} .
$$


Last, together with (5.15) for $y=x+1$, gives (5.17), as wanted.

Now, let $m_{x+1} \geq t_{x+1}$. Since (5.13) is valid, by Lemma 2.2. we have that for $u \in\{0, \ldots, s\}$ such that $h_{u}<z_{x+1}+t_{x+1} \leq h_{u+1}$, we have

$$
\sum_{i=z_{x+1}+t_{x+1}}^{m+s} g_{i} \geq \sum_{i=z_{x+1}+t_{x+1}-u}^{m} d_{i}+\sum_{i=u+1}^{s} a_{i}
$$

Now, by (5.14), (5.16) and (5.23), follow

$$
\sum_{i=x+1}^{h} c^{i}+\sum_{\substack{i>z_{x+1} \\ i \in \Delta}} d_{i} \geq \sum_{i=z_{x+1}+t_{x+1}}^{m+s} g_{i} \geq \sum_{i=z_{x+1}+t_{x+1}-u}^{m} d_{i}+\sum_{i=u+1}^{s} a_{i}
$$

Let $\left(e_{z_{x+1}+t_{x+1}}, \ldots, e_{z_{x+1}+m_{x+1}}\right)$ be the smallest $m_{x+1}-t_{x+1}+1$ elements among $\left(a_{1}, \ldots, a_{m_{x+1}}, d_{1}, \ldots, d_{z_{x+1}}\right)$.

Now we shall consider the possible values of $u$. We have three cases:

If $u<t_{x+1} \leq m_{x+1}$, then (5.24) gives

(5.25) $\sum_{i=x+1}^{h} c^{i}-\sum_{\substack{i>z_{x+1} \\ i \notin \Delta}} d_{i}-\sum_{i=m_{x+1}+1}^{s} a_{i} \geq-\sum_{i=z_{x+1}+1}^{z_{x+1}+t_{x+1}-u-1} d_{i}+\sum_{i=u+1}^{m_{x+1}} a_{i}$.

In this case, we have that

$$
\begin{gathered}
a_{u+1} \geq \cdots \geq a_{m_{x+1}-t_{x+1}+u+1} \geq \cdots \geq a_{m_{x+1}} \geq c^{x+1} \\
>d_{z_{x+1}+1} \geq \cdots \geq d_{z_{x+1}+t_{x+1}-u-1} .
\end{gathered}
$$

Hence, (5.25) implies

$$
\sum_{i=x+1}^{h} c^{i}-\sum_{\substack{i>z_{x+1} \\ i \notin \Delta}} d_{i}-\sum_{i=m_{x+1}+1}^{s} a_{i} \geq \sum_{i=z_{x+1}+t_{x+1}}^{z_{x+1}+m_{x+1}} e_{i}
$$

If $u \geq m_{x+1} \geq t_{x+1}$, then (5.24) gives

$$
\sum_{i=x+1}^{h} c^{i}-\sum_{\substack{i>z_{x+1} \\ i \notin \Delta}} d_{i}-\sum_{i=m_{x+1}+1}^{s} a_{i} \geq \sum_{i=z_{x+1}+t_{x+1}-u}^{z_{x+1}} d_{i}-\sum_{i=m_{x+1}+1}^{u} a_{i}
$$


In this case, we have that

$$
d_{z_{x+1}+t_{x+1}-u} \geq \cdots \geq d_{z_{x+1}}>c^{x+1} \geq a_{m_{x+1}+1} \geq \cdots \geq a_{u} .
$$

Hence, (5.27) implies (5.26).

Finally, if $m_{x+1}>u \geq t_{x+1}$, then (5.24) becomes

$$
\sum_{i=x+1}^{h} c^{i}-\sum_{\substack{i>z_{x+1} \\ i \notin \Delta}} d_{i}-\sum_{i=m_{x+1}+1}^{s} a_{i} \geq \sum_{i=z_{x+1}+t_{x+1}-u}^{z_{x+1}} d_{i}+\sum_{i=u+1}^{m_{x+1}} a_{i} .
$$

Last, by the definition of $e_{z_{x+1}+t_{x+1}}, \ldots, e_{z_{x+1}+m_{x+1}}$ also implies (5.26).

Thus, we have proved that in all the cases (5.26) is valid.

From (5.26) and (5.15), by Lemma 4.7, we have that for all $i$ such that $d_{i} \in$ $\left(e_{z_{x+1}+t_{x+1}}, \ldots, \ldots, e_{z_{x+1}+m_{x+1}}\right)$, we have that $i \notin \Delta$.

Denote by $E=\left(e_{z_{x+1}+t_{x+1}}, \ldots, e_{z_{x+1}+m_{x+1}}\right)$. The rest of the proof is split into two cases.

Case 1. There are no $d_{i}$ 's in $E$. Then

$$
E=\left(a_{t_{x+1}}, \ldots, a_{m_{x+1}}\right)=\left(a_{t_{x}+1}, \ldots, a_{m_{x+1}}\right) .
$$

Then (5.26) gives

$$
\sum_{i=x+1}^{h} c^{i}-\sum_{\substack{i>z_{x+1} \\ i \notin \Delta}} d_{i}-\sum_{i=m_{x+1}+1}^{s} a_{i} \geq \sum_{i=t_{x}+1}^{m_{x+1}} a_{i}
$$

i.e., we have that (5.17) is valid, as wanted.

Case 2. There is $i \in\left\{1, \ldots, z_{x+1}\right\}$ such that $d_{i} \in E$. By the definition of $z_{x+1}$ and by the definition of $E$, this implies that $d_{z_{x+1}} \in E$. Thus, by Lemma 4.7, $z_{x+1} \notin \Delta$. Since $w_{x}=0$, we have $d_{z_{x+1}}>c^{x}$. Let $v \geq 0$ be such that $c^{x-v-1}>d_{z_{x+1}}>c^{x-v}$.

Then

$$
c^{x-v-1}>d_{z_{x+1}}>c^{x-v} \geq \cdots \geq c^{x} \geq c^{x+1} .
$$

Last, together with the fact that $z_{x+1} \notin \Delta$, by (3.2), gives

$$
c^{x-v}+\cdots+c^{x}+\sum_{i=x+1}^{h} c^{i} \geq d_{z_{x+1}}+\sum_{\substack{i>z_{x+1} \\ i \notin \Delta}} d_{i}+\sum_{i=t_{x}-v+1}^{s} a_{i}
$$


Moreover, since

$$
\sum_{\substack{i>z_{x+1} \\ i \notin \Delta}} d_{i}=\sum_{\substack{i>z_{x} \\ i \notin \Delta}} d_{i}
$$

and since $d_{z_{x+1}}>c^{x-v}$, equation (5.28) implies

$$
\sum_{i=x+1}^{h} c^{i}-\sum_{\substack{i>z_{x} \\ i \notin \Delta}} d_{i}-\sum_{i=t_{x}+1}^{s} a_{i} \geq \sum_{i=t_{x}-v+1}^{t_{x}} a_{i}-c^{x-v+1}-\cdots-c^{x}
$$

However, since $d_{z_{x+1}} \in E$, the number of $a_{i}$ 's in $E$ is at most $m_{x+1}-t_{x+1}$, and so $a_{t_{x+1}} \notin E$. Hence, $a_{t_{x+1}}$ is bigger than or equal to all the elements from $E$, i.e., $a_{t_{x}+1}=a_{t_{x+1}} \geq d_{z_{x+1}}>c^{x-v}$. Hence, (5.29) implies (5.17), as wanted.

Analogously, we obtain the dual result:

LEMMA 5.5. Let $\mathbf{a}, \mathbf{b}, \mathbf{c}$ and $\mathbf{d}$ be partitions as given in (2.29)-2.32). Let $\mathbf{g}$ be a partition such that

$$
\mathbf{g} \prec^{\prime}(\mathbf{c}, \mathbf{b}) .
$$

Let $x \in\left\{0, \ldots, h^{\prime}-1\right\}$.

Suppose that

$$
\begin{gathered}
d^{i} \geq g_{z_{i}^{\prime}+t_{i}^{\prime}}, \quad i \geq x+1, \\
\sum_{i=y+1}^{h^{\prime}} d^{i} \geq \sum_{\substack{c_{i}<d^{y} \\
i \notin S}} c_{i}+\sum_{i=t_{y}^{\prime}+1}^{k} b_{i}, \quad \text { for } \quad y \geq x+1,
\end{gathered}
$$

and that for all $c_{i}<d^{x+1}, i \in S$, the following holds

$$
c_{i} \geq g_{i+t_{w}^{\prime}}, \quad \text { where } w \text { is such that } \quad d^{w}>c_{i}>d^{w+1} .
$$

Then

$$
\sum_{i=x+1}^{h^{\prime}} d^{i} \geq \sum_{\substack{c_{i}<d^{x} \\ i \notin S}} c_{i}+\sum_{i=t_{x}^{\prime}+1}^{k} b_{i}
$$


6. Proof of the main result. In this section, we give a proof of Theorem 5.1 .

6.1. Necessity. Suppose that there exists a partition $\mathbf{g}$ such that (5.1) and (5.2) are valid. Then, by the definition of the generalized majorization, we have that $(i)$ is valid. So we are left with proving the necessity of the conditions $(i i)$ and $(i i i)$.

In fact, we shall prove even more, that apart from $(i i)$ and (iii), the following also hold

$$
\begin{aligned}
& c^{x} \geq g_{z_{x}+t_{x}}, \quad x=1, \ldots, h, \\
& d^{y} \geq g_{z_{y}^{\prime}+t_{y}^{\prime}}, \quad y=1, \ldots, h^{\prime} .
\end{aligned}
$$

The proof goes by induction on $x=0, \ldots, h$ and $y=0, \ldots, h^{\prime}$. We prove that the conditions $(i i),($ iii $),(6.1)$ and (6.2) are satisfied for all the elements from the set $X=\left\{c_{i} \mid i \in S\right\} \cup\left\{d_{j} \mid j \in \Delta\right\}$.

More precisely, denote and order the elements from the set $X$ in the following way: $f_{1} \geq \cdots \geq f_{h+h^{\prime}}$. Then we shall prove that for every $\alpha \in\left\{1, \ldots, h+h^{\prime}\right\}$ the following is valid: if $f_{\alpha}=c^{i}$ for some $i=1, \ldots, h$, then $(i i)$ and (6.1) are satisfied for $x=i$, and if $f_{\alpha}=d^{j}$ for some $j=1, \ldots, h^{\prime}$, then (iii) and (6.2) are satisfied for $y=j$.

Before proceeding we note that by Lemma 4.5, the condition (6.1) is equivalent to the following:

For all $y=1, \ldots, h^{\prime}$ and for all $c_{i}<d^{y}, i \in S$ the following is valid

$$
c_{i} \geq g_{i+t_{w}^{\prime}}, \quad \text { where } d^{w}>c_{i}>d^{w+1} .
$$

Also, by Lemma 4.6 the condition (6.2) is equivalent to the following:

For all $x=1, \ldots, h$ and for all $d_{i}<c^{x}, i \in \Delta$ the following is valid

$$
d_{i} \geq g_{i+t_{w}}, \quad \text { where } c^{w}>d_{i}>c^{w+1} .
$$

The proof goes by the induction on $w \in\left\{1, \ldots, h+h^{\prime}\right\}$, starting from $h+h^{\prime}$.

As the base of induction, consider $f_{h+h^{\prime}}$. Suppose that $f_{h+h^{\prime}}=c^{h}$. Then we need to prove the necessity of $(i i)$ and (6.1) for $x=h$. However, by the definition of $t_{h}$ and by Lemma 4.1, condition (ii) is trivially satisfied. Moreover, (6.1) for $x=h$ becomes

$$
c^{h} \geq g_{z_{h}+s} .
$$

Let $j \in\{0, \ldots, n\}$ be such that $c^{h}=c_{j}$. Hence, by Lemma 4.5 (since $t_{h}=s$ and $d^{h^{\prime}}>c_{j}$ ), (6.3) is equal to $c^{h} \geq g_{j+t_{h^{\prime}}^{\prime}}$, which follows by Lemma 5.3. as wanted. 
If $f_{h+h^{\prime}}=d^{h^{\prime}}$, then we are left with proving the necessity of (iii) and (6.2) for $y=h^{\prime}$. By the definition of $t_{h^{\prime}}^{\prime}$ and by Lemma 4.3, condition (iii) is trivially satisfied. Also, completely analogously as in the proof of (6.1) for $x=h$, by Lemma 4.6, we have that (6.2) for $y=h^{\prime}$ becomes $d^{h^{\prime}} \geq g_{j+t_{h}}$. Last follows by Lemma 5.2, as wanted.

Now, we pass to the induction step. Let $w \in\left\{1, \ldots, h+h^{\prime}-1\right\}$. We are left with proving that if the conditions are satisfied for all $f_{j}$ with $j \in\left\{w+1, \ldots, h+h^{\prime}\right\}$, then they will be satisfied for $f_{w}$, as well. This is equivalent to the following:

If $f_{w}=c^{i}$, for some $i=1, \ldots, h$, and if the conditions (ii) and (6.1) are valid for all $x=i+1, \ldots, h$, and the conditions $(i i i)$ and (6.2) are valid for all $y$ such that $d^{y}<c^{i}$, then we are left with proving that the conditions (ii) and (6.1) are also valid for $x=i$.

In order to prove $(i i)$, it is enough to apply the result of Lemma 5.4, As for (6.1), let $u \in\left\{0, \ldots, h^{\prime}\right\}$ be such that

$$
d^{u}>c^{i}>d^{u+1}
$$

Then by the induction hypothesis, we have that (6.2) and (iii) are satisfied for all $y=u+1, \ldots, h^{\prime}$, and that (6.1) is satisfied for all $j \in S$ such that $c_{j}<d^{u+1}$. So, by applying Lemma 5.5 we have that the condition (iii) is satisfied for $y=u$ as well. Hence, we can now apply Lemma [5.3, and obtain (6.1) for $x=i$, as wanted.

Analogously as above, by the symmetry of the sets $S$ and $\Delta$ and by applying Lemmas 5.2, 5.4 and 5.5, we obtain that if $f_{w}=d^{i}$, for some $i=1, \ldots, h^{\prime}$, and if the conditions ( iii) and (6.2) are valid for all $y=i+1, \ldots, h$, and the conditions $(i i)$ and (6.1) are valid for all $x$ such that $c^{x}<d^{i}$, then the conditions (iii) and (6.2) are valid for $y=i$, as wanted.

Finally we are left with proving that the condition $(i i)$ is satisfied for $x=0$, and that (iii) is satisfied for $y=0$. The first follows by Lemma 5.4 for $x=0$, while the second follows by Lemma 5.5 for $x=0$.

This ends the proof of the necessity of the conditions $(i),(i i)$ and $(i i i)$.

6.2. Sufficiency. Let us suppose that the conditions $(i),(i i)$ and $(i i i)$ are satisfied.

We need to define a certain partition $\mathbf{g}$ such that (5.1) and (5.2) are valid.

We shall do this in two steps. First we are going to define a partition $\overline{\mathbf{g}}=$ $\left(\bar{g}_{1}, \ldots, \bar{g}_{m+s}\right)$ that satisfies

$$
d_{i} \geq \bar{g}_{i+s}, \quad i=1, \ldots, m,
$$




$$
c_{i} \geq \bar{g}_{i+k}, \quad i=1, \ldots, n,
$$

$$
\sum_{i=\bar{h}_{j}+1}^{m+s} \bar{g}_{i} \geq \sum_{i=\bar{h}_{j}-j+1}^{m} d_{i}+\sum_{i=j+1}^{s} a_{i}, \quad j=1, \ldots, s,
$$

where $\bar{h}_{j}=\min \left\{i \mid d_{i-j+1}<\bar{g}_{i}\right\}$,

$$
\sum_{i=\bar{h}_{j}^{\prime}+1}^{n+k} \bar{g}_{i} \geq \sum_{i=\bar{h}_{j}^{\prime}-j+1}^{n} c_{i}+\sum_{i=j+1}^{k} b_{i}, \quad j=1, \ldots, k,
$$

where $\bar{h}_{j}^{\prime}=\min \left\{i \mid c_{i-j+1}<\bar{g}_{i}\right\}$, and

$$
\sum_{i=1}^{m+s} \bar{g}_{i} \geq \sum_{i=1}^{m} d_{i}+\sum_{i=1}^{s} a_{i} .
$$

Then we shall define the wanted partition $\mathbf{g}$, by decreasing the elements of $\overline{\mathbf{g}}$ so that we obtain the correct sum, while preserving the remaining properties of the generalized majorization.

From the definition of $t_{0}$ and $t_{0}^{\prime}$, we have $t_{0}=t_{0}^{\prime}=m+s-h-h^{\prime}$. By Lemma 4.13. $t_{0} \geq 0$, and we define

$$
\bar{g}_{j}:=\max \left(d_{1}, a_{1}, c_{1}\right)+1, \quad j=1, \ldots, t_{0} .
$$

The remaining $h+h^{\prime}$ of $\bar{g}_{i}$ 's, i.e., $\bar{g}_{t_{0}+1}, \ldots, \bar{g}_{m+s}$, we define as a nonincreasing ordering of all $c_{i}$ 's with $i \in S$ and of all $d_{j}$ 's with $j \in \Delta$.

We can write this explicitly in the following way, by using the definitions of $z_{i}, t_{i}$ and the property (4.2) on the placement of $d_{j}$ 's with $j \in \Delta$ :

$$
\bar{g}_{j}:=\max \left(d_{1}, a_{1}, c_{1}\right)+1, \quad j=1, \ldots, t_{0},
$$

(6.11) $\bar{g}_{z_{i}+t_{i}}:=c^{i}, \quad i=1, \ldots, h$.

Recall that by Lemma 4.9 the sequence $z_{i}+t_{i}, i=0, \ldots, h$, is strictly increasing, and thus (6.10) and (6.11) are well-defined.

Dually, we can also write the explicit formula for $\bar{g}_{i}$ 's by exchanging the roles of $c_{i}$ 's and $d_{j}$ 's:

$$
\begin{aligned}
\bar{g}_{j} & :=\max \left(d_{1}, a_{1}, c_{1}\right)+1, \quad j=1, \ldots, t_{0}^{\prime}, \\
\text { (6.12) } \bar{g}_{j} & :=c_{j-t_{i}^{\prime}}, \quad z_{i}^{\prime}+t_{i}^{\prime}<j<z_{i+1}^{\prime}+t_{i+1}^{\prime}, \text { for } \\
\text { (6.13) } \bar{g}_{z_{i}^{\prime}+t_{i}^{\prime}} & :=d^{i}, \quad i=1, \ldots, h^{\prime} .
\end{aligned}
$$

Recall that by Lemma 4.10 the sequence $z_{i}^{\prime}+t_{i}^{\prime}, i=0, \ldots, h^{\prime}$, is strictly increasing, and thus (6.12) and (6.13) are well-defined. 
Proof of (6.4). By Lemma 4.18, we have that $t_{0} \leq s$, and so $\bar{g}_{j}$ 's involved in (6.4) are the ones defined by (6.10) and (6.11). From the definition, all such $\bar{g}_{j}$ 's satisfy

$$
\bar{g}_{j} \leq d_{j-t_{i}}, \quad \text { where } i \text { is such that } z_{i}+t_{i} \leq j<z_{i+1}+t_{i+1} .
$$

Finally, since $t_{i} \leq s$ (Lemma 4.18), for all $i=0, \ldots, h$, (6.14) implies (6.4), as wanted.

Proof of (6.5). Equation (6.5) follows analogously as (6.4), by duality and by Lemma 4.19.

Proof of ([6.6). From the definitions of $\bar{g}_{1} \geq \cdots \geq \bar{g}_{m+k}$, we have that

$$
\begin{aligned}
& \bar{h}_{j}=j, \quad j=1, \ldots, t_{0}, \\
& \bar{h}_{j}=z_{u}+t_{u}, \quad j=t_{0}+1, \ldots, s
\end{aligned}
$$

where $u:=\min \left\{i \in\{1, \ldots, h\} \mid t_{i}=j\right\}$ (note that $u$ is well-defined by Lemma 4.15).

Indeed, (6.15) follows from the definition (6.9). As for (6.16), first note that $\bar{h}_{j}$, for some $j \in\left\{t_{0}+1, \ldots, s\right\}$ is always equal to $z_{i}+t_{i}$, for some $i \in\{1, \ldots, h\}$. Otherwise, let $i \in\{0, \ldots, h\}$ be such that $z_{i}+t_{i}<\bar{h}_{j}<z_{i+1}+t_{i+1}$. Then by (6.10), we have $\bar{g}_{\bar{h}_{j}}=d_{\bar{h}_{j}-t_{i}}$, and from the definition of $\bar{h}_{j}$, we have $d_{\bar{h}_{j}-j+1}<\bar{g}_{\bar{h}_{j}}=d_{\bar{h}_{j}-t_{i}}$, which implies $j \leq t_{i}$, and so $i \geq 1$. But then, from (6.11), $\bar{g}_{z_{i}+t_{i}}=c^{i}>d_{z_{i}+1} \geq d_{z_{i}+t_{i}-j+1}$, and so $\bar{h}_{j} \leq z_{i}+t_{i}$.

Thus, for every $j \in\left\{t_{0}+1, \ldots, s\right\}, \bar{h}_{j}$ is of the form $z_{i}+t_{i}$, for some $i \in\{1, \ldots, h\}$. Finally, we claim that $\bar{h}_{j}=z_{u}+t_{u}$, where $u$ is the minimal index $i \in\{1, \ldots, h\}$ such that $t_{i}=j$. First of all, we have $\bar{g}_{z_{u}+t_{u}}=c^{u}>d_{z_{u}+1}=d_{z_{u}+t_{u}-j+1}$. Moreover, for all $\alpha<z_{u}+t_{u}$, we have that $\bar{g}_{\alpha} \leq d_{\alpha-j+1}$. Indeed, as shown in the previous paragraph it is enough to prove this fact for $\alpha$ of the form $z_{i}+t_{i}$, and since $\alpha<z_{u}+t_{u}$, we are left with proving $\bar{g}_{z_{\beta}+t_{\beta}} \leq d_{z_{\beta}+t_{\beta}-j+1}$, for $\beta<u$. From the definition of $u$ and since $t_{i+1} \leq t_{i}+1$, for all $i \in\{0, \ldots, h-1\}$, we have that $t_{\beta}<j$, for all $\beta<u$. Therefore $\bar{g}_{z_{\beta}+t_{\beta}}=c^{\beta} \leq d_{z_{\beta}} \leq d_{z_{\beta}+t_{\beta}-j+1}$, for all $\beta<u$, as wanted.

Hence, we have $\bar{h}_{j}=z_{u}+t_{u}$, where $u=\min \left\{i \in\{1, \ldots, h\} \mid t_{i}=j\right\}$, as wanted.

Now, the proof of (6.6) is split into two cases, depending on the value of $\bar{h}_{j}$, $j=1, \ldots, s$.

Case $j=1, \ldots, t_{0}$ : Then $\bar{h}_{j}=j$, and the condition (6.6) becomes

$$
\sum_{i=j+1}^{m+s} \bar{g}_{i} \geq \sum_{i=1}^{m} d_{i}+\sum_{i=j+1}^{s} a_{i}, \quad j=1, \ldots, t_{0}
$$


The condition $(i i)$ for $x=0$ gives

$$
\sum_{i=1}^{h} c^{i} \geq \sum_{i \notin \Delta} d_{i}+\sum_{i=t_{0}+1}^{s} a_{i}
$$

i.e.,

$$
\sum_{i=1}^{h} c^{i}+\sum_{j=1}^{h^{\prime}} d^{j} \geq \sum_{i=1}^{m} d_{i}+\sum_{i=t_{0}+1}^{s} a_{i}
$$

Hence, we obtain

$$
\sum_{i=t_{0}+1}^{m+s} \bar{g}_{i} \geq \sum_{i=1}^{m} d_{i}+\sum_{i=t_{0}+1}^{s} a_{i}
$$

Since $\bar{g}_{t_{0}} \geq a_{1}$, last gives (6.17), as wanted.

Case $j=t_{0}+1, \ldots, s$ : Then $\bar{h}_{j}=z_{u}+t_{u}=z_{u}+j$, where $u \in\{1, \ldots, h\}$ is the minimal index such that $t_{u}=j$. To prove (6.6), we are going to prove

$$
\sum_{i=z_{x}+t_{x}+1}^{m+s} \bar{g}_{i} \geq \sum_{i=z_{x}+1}^{m} d_{i}+\sum_{i=t_{x}+1}^{s} a_{i}, \quad \text { for all } x=1, \ldots, h .
$$

By the definition of $\bar{g}_{i}$ 's, we have that

$$
\sum_{i=z_{x}+t_{x}+1}^{m+s} \bar{g}_{i}=\sum_{i=z_{x}+1}^{m} d_{i}-\sum_{\substack{i>z_{x} \\ i \notin \Delta}} d_{i}+\sum_{i=x+1}^{h} c^{i} .
$$

Thus, we are left with proving

$$
\sum_{i=x+1}^{h} c^{i} \geq \sum_{\substack{d_{i}<c^{x} \\ i \notin \Delta}} d_{i}+\sum_{i=t_{x}+1}^{s} a_{i}, \quad x=1, \ldots, h .
$$

Last is exactly the condition $(i i)$, which finishes the proof.

Proof of (6.7). Equation (6.7) follows analogously as (6.6) by the symmetry of the sets $S$ and $\Delta$, i.e., by duality between $c_{i}$ 's and $d_{i}$ 's, and by the condition (iii).

Proof of (6.8). Again, from the condition (ii) for $x=0$ and the definition of $\bar{g}_{i}$ 's, we have:

$$
\sum_{i=t_{0}+1}^{m+s} \bar{g}_{i} \geq \sum_{i=1}^{m} d_{i}+\sum_{i=t_{0}+1}^{s} a_{i}
$$


Equation (6.18), together with $\bar{g}_{t_{0}} \geq a_{1}$, gives

$$
\sum_{i=1}^{m+s} \bar{g}_{i} \geq \sum_{i=1}^{m} d_{i}+\sum_{i=1}^{s} a_{i}
$$

as wanted.

Now, our aim is to decrease some of the $\bar{g}_{i}$ 's in order to define $g_{i}$ 's which will satisfy

$$
d_{i} \geq g_{i+s}, \quad i=1, \ldots, m
$$$$
c_{i} \geq g_{i+k}, \quad i=1, \ldots, n,
$$

$$
\sum_{i=h_{j}+1}^{m+s} g_{i} \geq \sum_{i=h_{j}-j+1}^{m} d_{i}+\sum_{i=j+1}^{s} a_{i}, \quad j=1, \ldots, s,
$$

where $h_{j}=\min \left\{i \mid d_{i-j+1}<g_{i}\right\}$,

$$
\sum_{i=h_{j}^{\prime}+1}^{n+k} g_{i} \geq \sum_{i=h_{j}^{\prime}-j+1}^{n} c_{i}+\sum_{i=j+1}^{k} b_{i}, \quad j=1, \ldots, k,
$$

where $h_{j}^{\prime}=\min \left\{i \mid c_{i-j+1}<g_{i}\right\}$, and

$$
\sum_{i=1}^{m+s} g_{i}=\sum_{i=1}^{m} d_{i}+\sum_{i=1}^{s} a_{i}
$$

We shall do this in the following way: start from $\bar{g}_{1}$ and decrease it till $\bar{g}_{2}$. If the sum is OK, stop. If not proceed by decreasing $\bar{g}_{1}$ and $\bar{g}_{2}$ till $\bar{g}_{3}$. And so on until we have decreased $\bar{g}_{i}$ 's such that (6.23) is valid.

More precisely, let $\Omega:=\sum_{i=1}^{m+s} \bar{g}_{i}-\left(\sum_{i=1}^{s} a_{i}+\sum_{i=1}^{m} d_{i}\right) \geq 0$ and let $f:=$ $\min \left\{i \mid \sum_{j=1}^{i} \bar{g}_{j}-i \bar{g}_{i} \geq \Omega\right\}$. Then we are going to define $g_{i}, i=1, \ldots, m+s$, such that

$$
\begin{aligned}
& \sum_{i=1}^{m+s} g_{i}=\sum_{i=1}^{m} d_{i}+\sum_{i=1}^{s} a_{i} \\
& g_{i}=\bar{g}_{i}, \quad \text { for all } \quad i \geq f, \\
& \bar{g}_{f-1} \geq g_{i} \geq \bar{g}_{f} \quad \text { for all } \quad i=1, \ldots, f-1,
\end{aligned}
$$


and

$$
g_{1} \geq g_{f-1} \geq g_{1}-1
$$

In other words, we decrease the smallest possible number of $\bar{g}_{i}$ 's, such that the sum is correct, and such that $g_{1} \geq g_{2} \geq \ldots \geq g_{f-1}$ becomes the most homogeneous partition of $\bar{g}_{1}+\bar{g}_{2}+\cdots+\bar{g}_{f-1}-\Omega$. Such defined $g_{1} \geq \cdots \geq g_{m+s}$ satisfy (6.23).

Since $\bar{g}_{i} \geq g_{i}, i=1, \ldots, m+s$, from (6.4) and (6.5), we have that (6.19) and (6.20) are valid. So we are left with proving (6.21) and (6.22).

Proof of (6.21). Follows directly by applying Lemma 2.4 for $\overline{\mathbf{g}}, \mathbf{d}, \mathbf{a}, f$ and $\mathbf{g}$.

Proof of (6.22). Follows by Lemma 2.4 for $\overline{\mathbf{g}}, \mathbf{c}, \mathbf{b}, f$ and $\mathbf{g}$.

Now, conditions (6.19), (6.21) and (6.23) give (5.1), while conditions (6.20), (6.22) and (6.23) give (5.2). This finishes our proof. $\mathrm{C}$

7. Some corollaries. In course of the proof of Theorem 5.1 we have in fact obtained a solution for the analogous slightly relaxed problem. Namely, we can define a weak generalized majorization:

Definition 7.1. Let $\mathbf{a}=\left(a_{1}, \ldots, a_{s}\right), \mathbf{d}=\left(d_{1}, \ldots, d_{m}\right)$ and $\mathbf{g}=\left(g_{1}, \ldots, g_{m+s}\right)$ be nonincreasing partitions. We write

$$
\mathbf{g} \prec^{\prime \prime}(\mathbf{d}, \mathbf{a})
$$

if the following three conditions are satisfied:

$$
\begin{gathered}
d_{i} \geq g_{i+s}, \quad i=1, \ldots, m, \\
\sum_{i=h_{j}+1}^{m+s} g_{i}-\sum_{i=h_{j}-j+1}^{m} d_{i} \geq \sum_{i=j+1}^{s} a_{i}, \quad j=1, \ldots, s \\
\sum_{i=1}^{m+s} g_{i} \geq \sum_{i=1}^{m} d_{i}+\sum_{i=1}^{s} a_{i}
\end{gathered}
$$

where $h_{j}:=\min \left\{i \mid d_{i-j+1}<g_{i}\right\}, j=1, \ldots, s$.

Then by repeating the same proof as for Theorem 5.1, we have:

THEOREM 7.2. Let $\mathbf{a}, \mathbf{b}, \mathbf{c}$ and $\mathbf{d}$ be partitions as given in (2.29)-(2.32).

There exists a partition $\mathbf{g}=\left(g_{1}, \ldots, g_{m+s}\right)$, such that

$$
\begin{aligned}
& \mathrm{g} \prec^{\prime \prime}(\mathbf{d}, \mathbf{a}) \\
& \mathrm{g} \prec^{\prime \prime}(\mathbf{c}, \mathbf{b})
\end{aligned}
$$


if and only if the following conditions are valid:

$$
\begin{aligned}
& \text { (i) } \sum_{i=x+1}^{h} c^{i} \geq \sum_{\substack{d_{i}<c^{x} \\
i \notin \Delta}} d_{i}+\sum_{i=t_{x}+1}^{s} a_{i}, \quad x=0, \ldots, h, \\
& \text { (ii) } \sum_{i=y+1}^{h^{\prime}} d^{i} \geq \sum_{\substack{c_{i}<d^{y} \\
i \notin S}} c_{i}+\sum_{i=t_{y}^{\prime}+1}^{k} b_{i}, \quad y=0, \ldots, h^{\prime} .
\end{aligned}
$$

Recall that by the result obtained in Subsection 2.2, in Theorem 7.2 we are only considering partitions $\mathbf{c}$ and $\mathbf{d}$ such that for all $i=1, \ldots, n$, and for all $j=1, \ldots, m$, we have $c_{i} \neq d_{j}$.

7.1. Nonnegative partitions. In this subsection, we are considering Problem 1, in a case when the involved partitions consist only of nonnegative integers. In this case, some improvements can be made in Theorem 5.1. Also, this restriction is particularly important in the applications in the matrix and matrix pencils completion problems.

Let $\mathbf{c}=\left(c_{1}, \ldots, c_{n}\right)$ and $\mathbf{d}=\left(d_{1}, \ldots, d_{m}\right)$ be partitions such that $c_{1} \geq \cdots \geq c_{c}>$ $c_{c+1}=\cdots=c_{n}=0$ and $d_{1} \geq \cdots \geq d_{d}>d_{d+1}=\cdots=d_{m}=0$. Then we can determine the number of nonzero elements of partitions $\overline{\mathbf{g}}$ and $\mathbf{g}$ obtained as in the sufficiency part of the proof of Theorem 5.1 (Section 6.2).

Namely, let $\overline{\mathbf{g}}=\left(\bar{g}_{1}, \ldots, \bar{g}_{m+s}\right)$, where $\bar{g}_{1} \geq \cdots \geq \bar{g}_{\bar{g}}>\bar{g}_{\bar{g}+1}=\cdots=\bar{g}_{m+s}=0$ be defined as in (6.9)-6.11). Then,

$$
m+s-\bar{g}=\max (n-c, m-d) .
$$

Indeed, if both $c_{i}$ 's and $d_{j}$ 's contain zeros (i.e., if $c<n$ and $d<m$ ), then by Proposition 2.6 we can "erase" the same number of zeros from both of them. So, without loss of generality, we can assume that only one of the partitions $\mathbf{c}$ or $\mathbf{d}$ contains zeros, say $\mathbf{d}$. Then from the definition of the set $\Delta$, all $j=d+1, \ldots, m$ satisfy $j \in \Delta$ (since for all of them $q_{j}=s+1$ ). Since $\left\{\bar{g}_{i}\right\}_{i=t_{0}+1}^{m+s}=\left\{c_{i} \mid i \in S\right\} \cup\left\{d_{j} \mid j \in \Delta\right\}$, we have that $m+s-\bar{g}=m-d$, as wanted. We get the analogous result in the case when only partition c contains zeros. Altogether, we have proved (7.1), as wanted.

Equation (7.1) gives

$$
\bar{g}=\min (c+k, d+s)
$$

Now, from the definition of $\mathbf{g}=\left(g_{1}, \ldots, g_{m+s}\right)$ with $g_{1} \geq \cdots \geq g_{g}>g_{g+1}=$ $\cdots=g_{m+s}=0$, we have that either $g=\bar{g}$, or we have that $g_{1}=\cdots=g_{g}=1$, 
in which case $g \leq \bar{g}$. In the latter case we have $g=\sum_{i=1}^{m+s} g_{i}=\sum_{i=1}^{m} d_{i}+\sum_{i=1}^{s} a_{i}$ $=\sum_{i=1}^{n} c_{i}+\sum_{i=1}^{k} b_{i}$, and thus we obtain:

Proposition 7.3. Let $\mathbf{c}=\left(c_{1}, \ldots, c_{n}\right)$ and $\mathbf{d}=\left(d_{1}, \ldots, d_{m}\right)$ be nonnegative partitions with $c_{1} \geq \cdots \geq c_{c}>c_{c+1}=\cdots=c_{n}=0$ and $d_{1} \geq \cdots \geq d_{d}>d_{d+1}=$ $\cdots=d_{m}=0$. Let $\mathbf{g}=\left(g_{1}, \ldots, g_{m+s}\right)$ be partition defined in the sufficiency part of Theorem 5.1 in Section 6.2. Then $g_{1} \geq \cdots \geq g_{g}>g_{g+1}=\cdots=g_{m+s}=0$ with

$$
g=\min \left(c+k, d+s, \sum_{i=1}^{m} d_{i}+\sum_{i=1}^{s} a_{i}\right) .
$$

Acknowledgment. The authors would like to thank the referee for hers/his suggestions and comments. This work was done within the activities of CELC and was partially supported by FCT, project ISFL-1-1431, and by the Ministry of Science of Serbia, projects no. 174020 (M.D.) and no. 174012 (M.S.).

\section{REFERENCES}

[1] I. Baragaña and I. Zaballa. Column completion of a pair of matrices. Linear Multilinear Algebra, 27:243-273, 1990.

[2] I. Baragaña and I. Zaballa. Feedback invariants of restrictions and quotiens: Series connected systems. Linear Algebra Appl., 351/352:69-89, 2002.

[3] R. Bhatia. Matrix Analysis. Springer, 1996.

[4] M. Dodig. Matrix pencils completion problems. Linear Algebra Appl., 428(1):259-304, 2008.

[5] M. Dodig. Rank distance problem for pairs of matrices. Linear Multilinear Algebra, 61:205-215, 2013.

[6] M. Dodig. Completion up to a matrix pencil with column minimal indices as the only nontrivial Kronecker invariants. Linear Algebra Appl., 438:3155-3173, 2013.

[7] M. Dodig. Completion of quasi-regular matrix pencils. Submitted for publication.

[8] M. Dodig and M. Stošić. Combinatorics of column minimal indices and matrix pencil completion problems. SIAM J. Matrix Anal. Appl., 31:2318-2346, 2010.

[9] M. Dodig and M. Stošić. On convexity of polynomial paths and generalized majorizations. Electron. J. Combin., 17(1):R61, 2010.

[10] M. Dodig and M. Stošić. Applications of the generalized majorization. Preprint, 2012.

[11] I. Gohberg, M.A. Kaashoek, and F. van Schagen. Eigenvalues of completions of submatrices. Linear Multilinear Algebra, 25:55-70, 1989.

[12] G. Hardy, J.E. Littlewood, and G. Pólya. Inequalities. Cambridge University Press, Cambridge, 1991.

[13] J. Karamata. Sur une inégalité relative aux fonctions convexes. Publ. Math. Univ. Belgrade, 1:145-158, 1932.

[14] J. Loiseau, S. Mondié, I. Zaballa, and P. Zagalak. Assigning the Kronecker invariants of a matrix pencil by row or column completions. Linear Algebra and Appl., 278:327-336, 1998.

[15] S. Mondié. Contribution à l'Étude des Modifications Structurelles des Systèmes Linéares. PhD. Thesis, Université de Nantes, 1996.

[16] M. Nielsen and I. Chuang. Quantum Computation and Quantum Information. Cambridge University Press, Cambridge, 2000. 
Electronic Journal of Linear Algebra ISSN 1081-3810

A publication of the International Linear Algebra Society

Volume 26, pp. 471-509, July 2013

[17] H.H. Rosenbrock. State-Space and Multivariable Theory. Thomas Nelson and Sons, London, 1970.

[18] I. Zaballa. Matrices with prescribed rows and invariant factors. Linear Algebra Appl., 87:113$146,1987$. 